DOI: https://doi.org/10.24297/jam.v20i.9042

\title{
PDE boundary conditions that eliminate quantum weirdness: a mathematical game inspired by Kurt Gödel and Alan Turing
}

Author: Jeffrey H. Boyd ${ }^{*}$

Affiliation: * Retired. 57 Woods Road, Bethany, CT, 06524-3103 USA

Jeffrey.Boyd@alumni.harvard.edu

\begin{abstract}
:
Although boundary condition problems in quantum mathematics (QM) are well known, no one ever used boundary conditions technology to abolish quantum weirdness. We employ boundary conditions to build a mathematical game that is fun to learn, and by using it you will discover that quantum weirdness evaporates and vanishes. Our clever game is so designed that you can solve the boundary condition problems for a single point if-and-only-if you also solve the "weirdness" problem for all of quantum mathematics. Our approach differs radically from Dirichlet, Neumann, Robin, or Wolfram Alpha. We define domain $\Omega$ in one-dimension, on which a partial differential equation (PDE) is defined. Point $\boldsymbol{\alpha}$ on $\partial \Omega$ is the location of a boundary condition game that involves an off-center bi-directional wave solution called $\boldsymbol{E}$, an "elementary wave." Study of this unusual, complex wave is called the Theory of Elementary Waves (TEW). We are inspired by Kurt Gödel and Alan Turing who built mathematical games that demonstrated that axiomatization of all mathematics was impossible. In our machine quantum weirdness vanishes if understood from the perspective of a single point $\boldsymbol{\alpha}$, because that pinpoint teaches us that nature is organized differently than we expect.
\end{abstract}

Mathematics Subject Classification (MSC2010): 81Q65 Alternative Quantum Mechanics

Key words: mathematical enigmas and puzzles, unsolved problems, metaphysics, worldview

\section{Introduction}

Here is a puzzle you will enjoy. Why do quantum particles behave differently if we are watching them, than if we close our eyes? Richard Feynman, like other quantum experts, said this was true. But he couldn't explain it. This article will teach you a PDE boundary condition game, which will give you the tools to solve this puzzle. Hint: the puzzle tells you that the human eye is sending a signal to a quantum particle and the particle is responding. Can you write the simplest possible complex wave equations for one-dimension? This is a game.

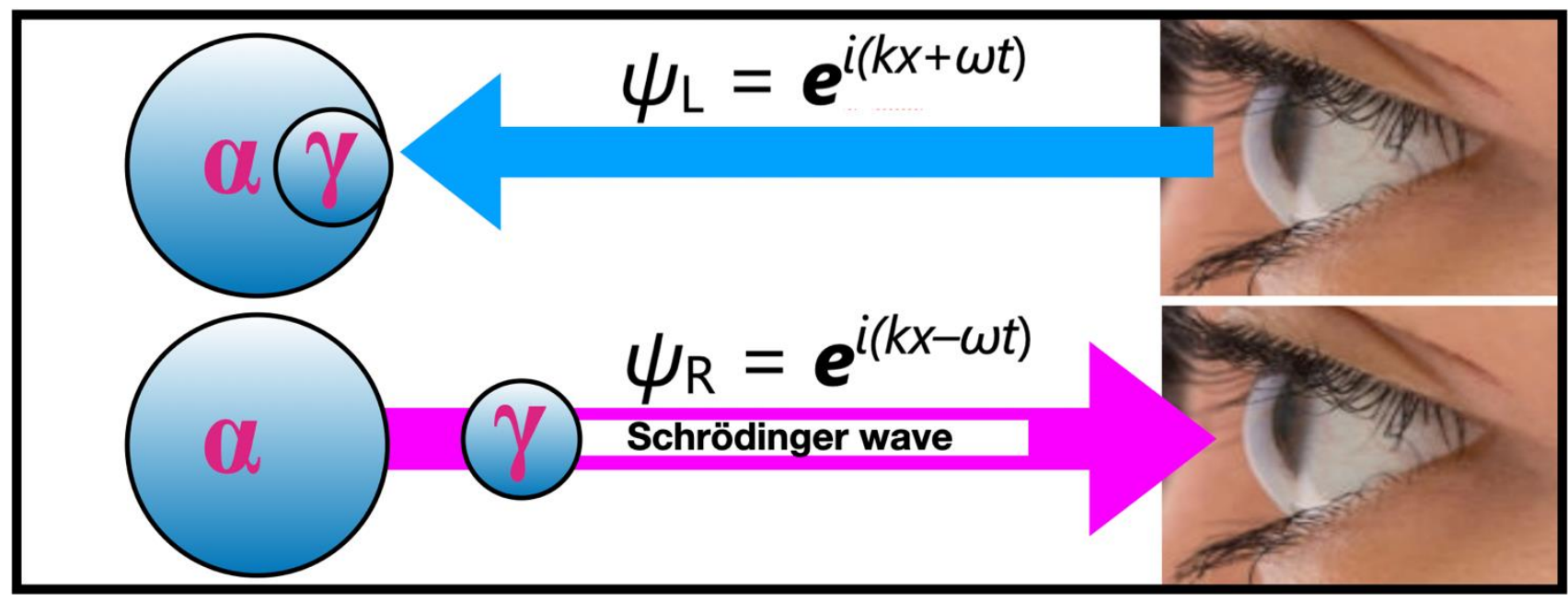

Fig. 1. Top: plane wave wave $\psi_{\mathrm{L}}$ emanates from your eye (the detector). At particle $\boldsymbol{\alpha}$ it reflects and becomes a Schrödinger wave carrying photon $\boldsymbol{\gamma}$ back to your eye. If you close your eye then $\psi$ L vanishes. Domain $\Omega$ is from particle $\boldsymbol{\alpha}$ to your eye. The complicated boundary condition is at point $\boldsymbol{\alpha}$ (where particle $\boldsymbol{\alpha}$ is located). 
In our game the detector (your eye) and the quantum object are interacting with each other, like dancing partners. When you close your eyes then wave $\psi$ L would abruptly vanish, which would mean that particle $\boldsymbol{\alpha}$ no longer interacts with wave $\psi_{\mathrm{L}}$. So it behaves differently. Perhaps it interacts with a wave coming from the wall beside you, but it is no longer connected to your eye. In our game we call these " $\psi$ " waves "elementary waves," and when we develop a system of math that we call the "Theory of Elementary Waves" (TEW). These would be zero-energy waves. (1,3-30,44-48,51,54-56)

In our model zero-energy waves exist. It is usually said that "all waves carry energy." (51) Some authorities define a "wave" by how it conveys energy. But quantum waves carry zero-energy. The Schrödinger wave is an example. It conveys a Hamiltonian operator or a momentum operator, but it carries no raw energy. The Schrödinger wave does not push or pull particles. It does no work. Schrödinger waves predict the future, but they do not thrust particles in the direction of that future. In our mechanism (Fig. 1) all energy and momentum are carried by the particle not by the waves.

The purpose of our gadget is to take the amorphous confusion called "quantum weirdness" and downsize it to a pinpoint named $\boldsymbol{\alpha}$. Although you might be intimidated by quantum weirdness, surely you are not intimidated by a pinpoint! Be bold! This is only make-believe. When we apply the well-known technology of PDE boundary conditions to that pinpoint we bring our subject matter into focus. That single point has something to teach us. Nature is organized in a way different than what people expect. When we introduce "elementary waves" into our mathematics of nature, weirdness evaporates and vanishes.

Our machine is interactive, like a dance. In Fig. 1-top you can see the domain $\Omega$ within which wave $\psi_{\mathrm{L}}$ is defined. In our one-dimensional model domain $\Omega$ extends from point $\boldsymbol{\alpha}$ to your eye. Particle $\boldsymbol{\alpha}$ has two states: reflective or transparent (Fig. 4). If it is reflective then it reflects $\psi_{\mathrm{L}}$ which becomes $\psi_{\mathrm{R}}$ and, as it reverses direction it becomes a Schrödinger wave carrying particle $\boldsymbol{\alpha}$, or a photon $\boldsymbol{\gamma}$ (i.e., energy from particle $\boldsymbol{\alpha}$ ) back to your eye. The domain of that Schrödinger wave $\left(\psi_{R}\right)$ is $\Omega$. The $\partial \Omega$ boundary is where particle $\boldsymbol{\alpha}$ resides.

You may be familiar with Dirichlet boundary condition that specifies the values that a PDE solution needs to take along the boundary of the domain. But you never heard of a boundary condition that says that "a complex plane wave $\psi\llcorner$ reflects off particle $\boldsymbol{\alpha}$ at the $\partial \Omega$ boundary, and as it reverses direction it becomes a Schrödinger wave named $\psi_{R}$ that transports particle $\boldsymbol{\alpha}$ back to the detector, and the detector is the other boundary in this onedimensional domain." This sentence is so dissimilar to Dirichlet that one wonders if it should be called a "boundary condition." But what else would you call it? It is a condition located at a domain boundary. $(31,53)$

The linear PDE defined over domain $\Omega$ is Equation 1 in our apparatus. The one-dimension is the $x$-axis, but " $u$ " is a complex function $u=\mathrm{e}^{\mathrm{i}(\mathrm{k} x \pm \omega t)}$. That 2-way-wave is a lot trickier than you would expect.

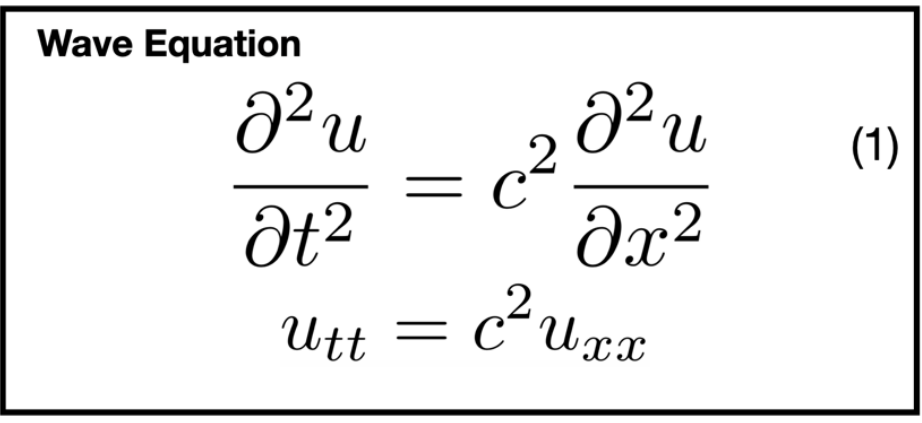

Our model differs from the behavior of most linear partial differential equations. Normally you would think that Eq. 1 would have two solutions that are equal but going in opposite directions [to the left $\left(\psi_{\mathrm{L}}=\boldsymbol{e}^{i(k x+\omega t)}\right)$, and to the right $\left.\left(\psi_{R}=\boldsymbol{e}^{i(k x-\omega t)}\right)\right]$. That would lead you to think these solutions can be added linearly $\left(A \psi_{L}+B \psi_{R}\right)$ to get another solution. However, in our game $\psi_{\mathrm{R}}$ is not a mirror image of $\psi_{\mathrm{L}}$. The wave to the right blossoms immediately from a plane into a Schrödinger wave and instantly acquires a particle $\boldsymbol{\alpha}$ to carry in its truck bed. In our arrangement $\psi_{\mathrm{L}}$ is incapable of becoming a Schrödinger wave. This is important because the whole purpose of playing our game is to teach you to understand the unique features of "point $\boldsymbol{\alpha}$." 
Since we cannot simply add the two solutions $\left[\left(A \psi_{L}+B \psi_{R}\right)\right]$ in the normal way, therefore we will need to invent another function called $\boldsymbol{E}$ as the name of this conglomerate $\left[A \psi_{L}+B \psi_{R}+\right.$ particle $\left.\boldsymbol{\alpha}\right]$ in which $\psi_{\mathrm{L}}$ is an ordinary plane wave whereas $\psi_{R}$ is a Schrödinger wave. The particle is not simply a wave packet. What you know as a "wave packet" is changed in our game into a truck bed that can carry a physical particle with weight and momentum. The particle( $\boldsymbol{\alpha})$ is not the same as the wave packet, just like a boulder is not the same as the bed of a dump truck. Our game is designed to teach us to understand things that are not simply "mathematics as usual."

Ours is not an "interpretation" of quantum math. Ours is a new mathematics. It is the mathematics of a hypothetical world called "The Elementary Wave World." We enfold the Schrödinger equation within other equations that change the meaning and behavior of a Schrödinger equation. In other words, the Schrödinger wave equation in our game is peculiar in its behavior, and you will be surprised at how different our Schrödinger wave equation behaves, than what you would expect.

You might say, "Yes, it is the Schrödinger equation (see Fig. 1 bottom), but it doesn't look or act like the Schrödinger equation." Usually, people think of a Schrödinger wave moving a particle from source to detector. In our model every Schrödinger wave had a pre-history. That pre-history started before the particle left the source. A plane wave $\psi_{\mathrm{L}}$ came from the detector, reflected off particle $\boldsymbol{\alpha}$ and metamorphized into a Schrödinger wave $\psi_{\mathrm{R}}$ carrying the particle back toward that detector. If you gloss over the details, that means that a particle moves towards a detector because it appears to be following backwards a zero-energy plane wave $\psi_{L}$ emanating out of the detector.

Our model uses only free particles. This machine does not change how bound particles are understood. The Periodic Table, chemistry, atoms, molecules, biochemistry, and harmonic oscillators are no different with TEW than with standard QM. Ours is a new perspective on the boundary conditions governing complex waves interacting with a single free particle in one dimension.

In our game there is only one free particle with no spin and no charge, in no electromagnetic field. Our particle looks and acts like cricket balls or baseball. In our hypothetical world, wave-particle duality is wrong. (1,3-30,4448,51,54-56)

\subsection{Another puzzle for you to solve}

Here is another riddle. Niels Bohr said that the final data from a quantum experiment are determined by the final arrangement of the detectors. If the detectors are rearranged while the particle is in flight, the particle somehow knows about it and adjusts accordingly. Building a logic to explain that quirk is one of the agendas of this article. That is like how the Turing machine was designed for the central agenda of solving a paradox in mathematical logic, namely the halting problem.

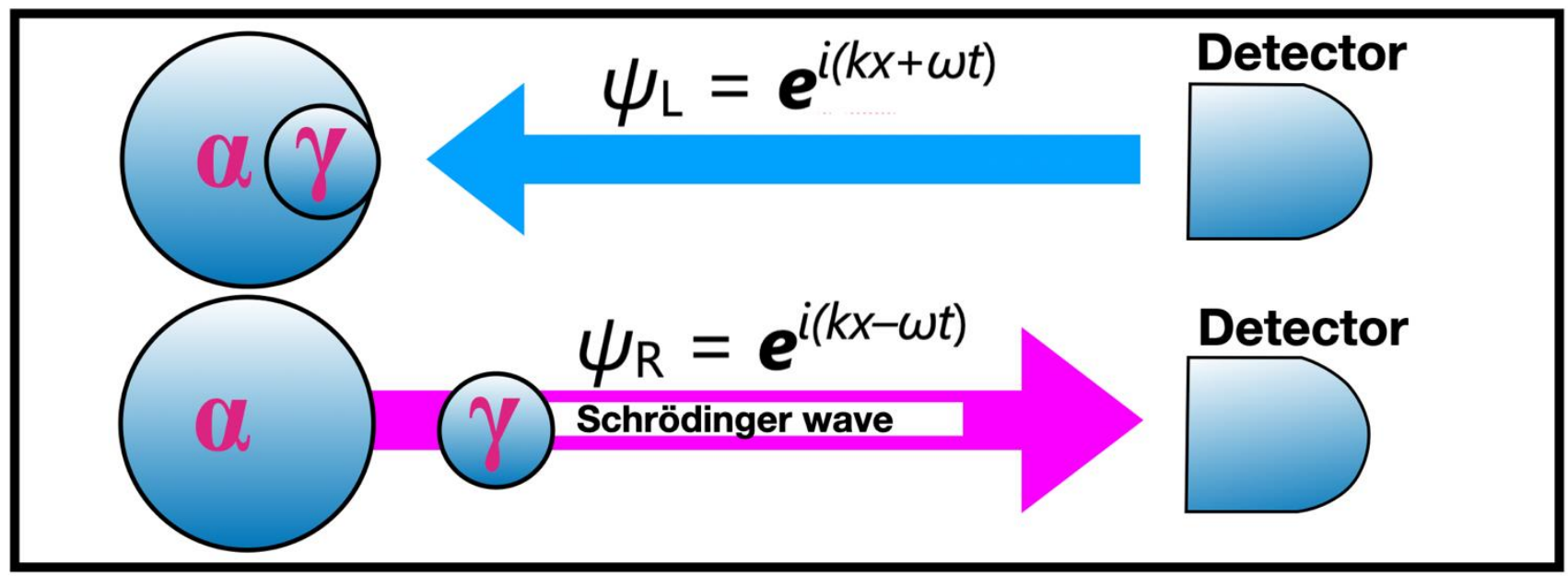

Fig. 2. The final data in a quantum experiment are determined by the final arrangement of the detectors. If the detectors are rearranged while the particle is in flight, the particle knows it and adjusts accordingly. 
Puzzle \#2 is just a repeat of puzzle \#1, but in puzzle \#2 particle $\boldsymbol{\alpha}$ is already in motion when it is intercepted by wave $\psi\llcorner$ shooting out from the detector.

\subsection{Puzzle \#3 - the Purcell effect}

The Purcell effect was discovered in 1946. A Rydberg atom (such as sodium, cesium, beryllium, magnesium, or calcium) is heated in an oven, then a laser excites the outer electron to a higher energy state, and the atom is injected into a microcavity. The outer electron will drop to a lower energy level and emit a photon 500 times faster if the cavity is resonant than if it is not. "Resonance" depends on something called the "mode of the cavity," which is also called an "available state." This is determined by whether the microcavity has a diameter equal to a multiple $\lambda / 2$, where $\lambda$ is the wavelength of the photon that would be emitted. $(41,52)$

How does a hot and excited Rydberg atom naively wandering into a cavity know the width of the cavity? Does a quantum wave from inside the atom venture out to measure the width of the environment, and then if the width is inhospitable the quantum wave goes back inside the atom and tells its electron, "Forget it"? No! Rather a "mode of the cavity" is something of zero energy that already lives inside the cavity before the Rydberg atom arrives.

We rename "modes of the cavity" and call them "elementary waves."

We rename "available states" and call them "elementary waves."

This demonstrates that physicists knew about elementary waves since 1946, but never developed a systematic science of elementary waves. The Purcell effect demonstrates how zero-energy waves can interact with an excited electron. If the width of the cavity is not perfect, the excited electron will remain excited and not drop to a lower energy orbit. The zero-energy elementary waves are in control. They are in control in the sense that they permit something to happen, using the energy intrinsic to the excited electron.

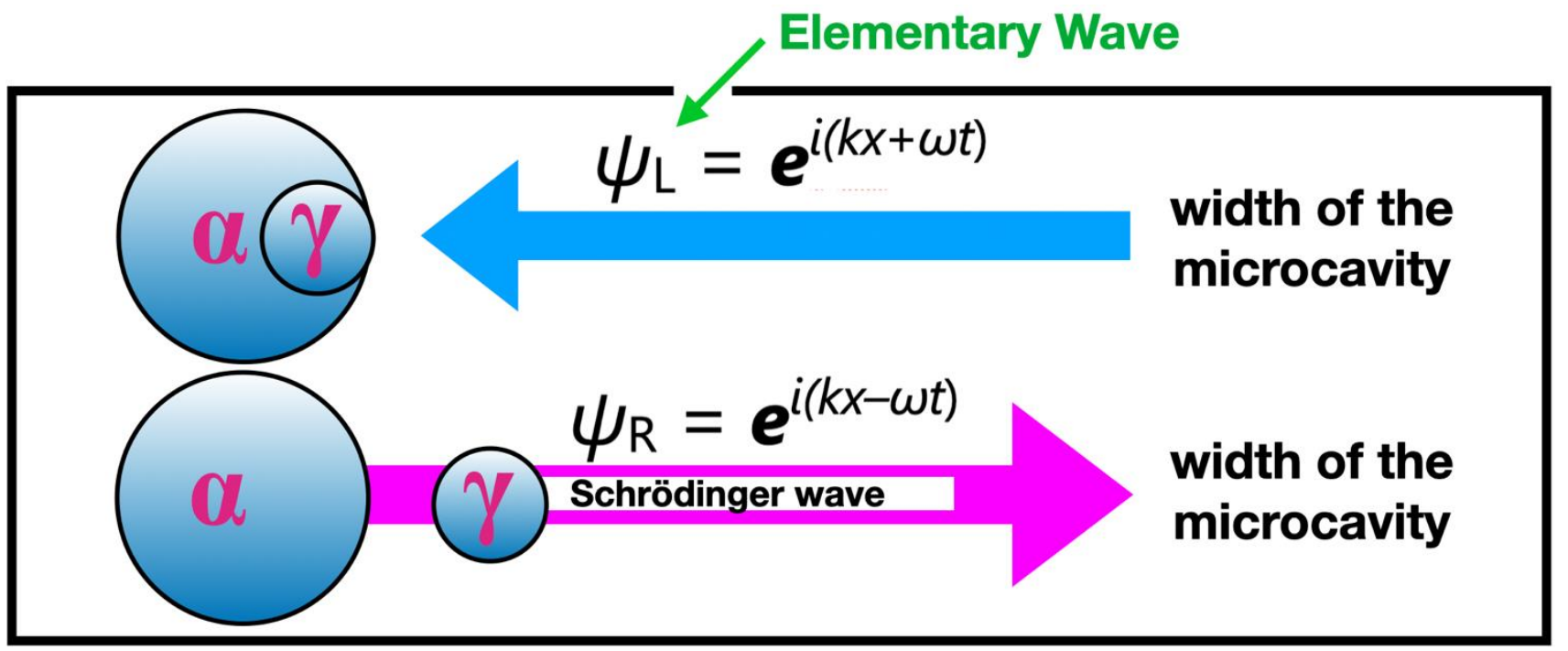

Fig. 3. The Purcell effect is when an excited outer electron $\boldsymbol{\alpha}$ in a Rydberg atom in a microcavity interacts with a "mode of the cavity" defined by the cavity's diameter. We rename the "mode of the cavity" and call it "elementary wave $\psi_{\text {L. }}$ 


\section{.3 Here is the mathematical game we will build together}

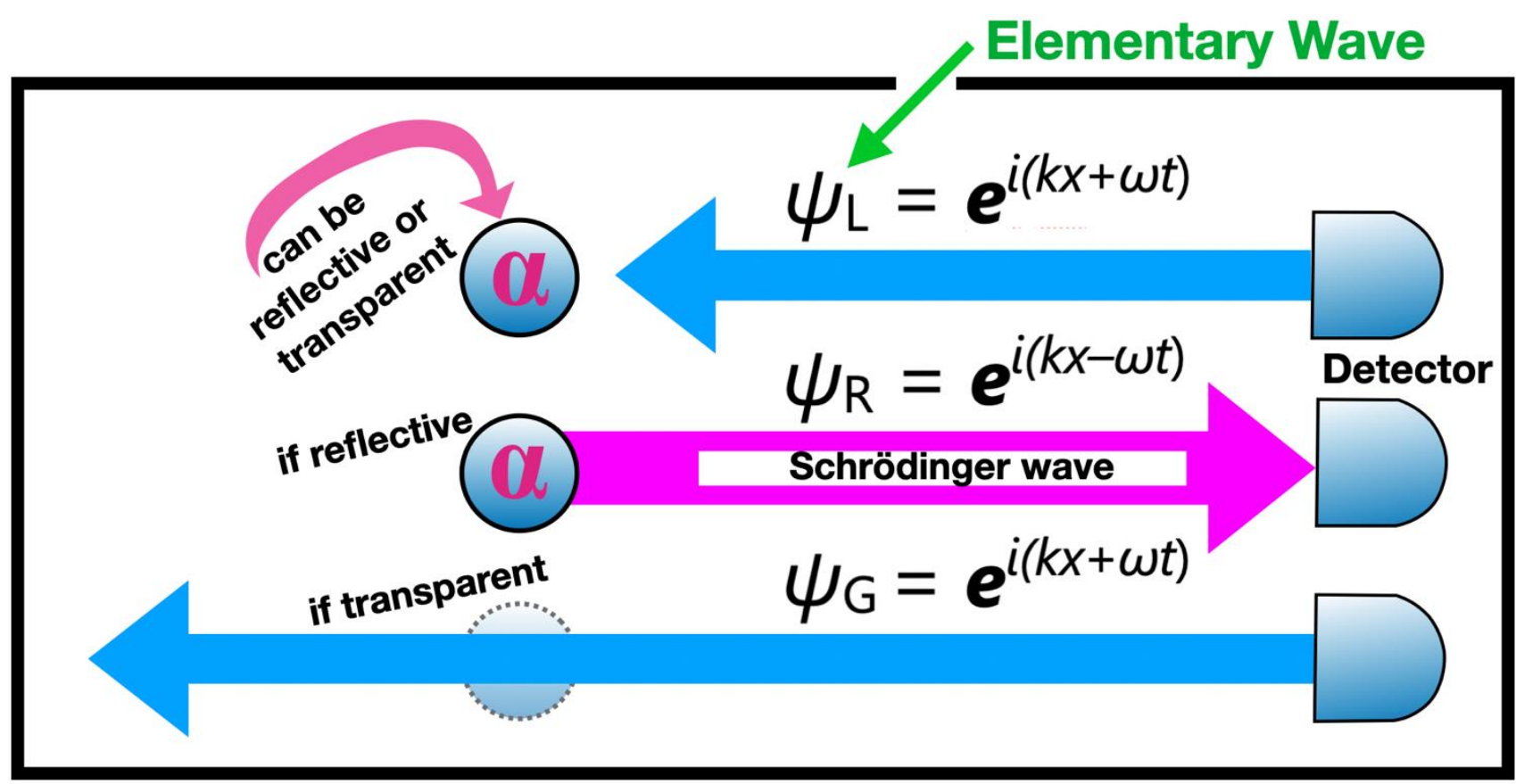

Fig. 4. This is our model or game. The particle named $\boldsymbol{\alpha}$ has two conditions and the wave named $\psi$ has three. If the particle chooses at random to be transparent, the wave $\psi$ L passes through and vanishes off the horizon. In that case we name it $\psi_{\mathrm{G}}$ where subscript " $G$ " stands for "Ghost" because our detectors can see particles but not waves. The waves in this Figure, especially the top one, are called "elementary waves."

As noted above, this article presents an unusual boundary condition discussion, aimed at building a game or mathematical machine. We will build a one-dimensional model in which there is a domain $\Omega$ over which the equation $\mathbf{u}_{\mathbf{t t}}=\mathbf{c}^{\mathbf{2}} \mathbf{u}_{\mathbf{x x}}$ is defined. What we call the "boundary condition" is the intricate engine (Fig. 4) we build at point $\boldsymbol{\alpha}$ where particle $\boldsymbol{\alpha}$ lives. That point might be stationary or moving along the axis. The boundary condition consists of the mandates on particle $\boldsymbol{\alpha}$ located at the pinpoint border at $\partial \Omega$. These mandates are pivotal to the transformation of $\psi_{\mathrm{L}}$ into $\psi_{\mathrm{R}}$ which becomes a Schrödinger wave carrying particle $\boldsymbol{\alpha}$ away toward the detector. That interaction, which is complicated and unexpected, is the "boundary condition" that, we will show, eliminates quantum weirdness. As we said before, if you think this should not be called a "boundary condition," then what would you call this condition focused on boundary $\partial \Omega$ ?

The reader may wonder why we are doing something so convoluted. Because, like Kurt Gödel and Alan Turing, we are designing a small hook to catch a big fish. Their hook (which was not boundary conditions) demolished David Hilbert's proposed axiomatization of Cantor's set theory, cardinal numbers, and all math.

Long story short, in this article we will invent, design, and assemble a mathematical machine or game with unique boundary condition rules that are fun to learn, that will hopefully persuade you to rethink quantum weirdness. If you play around with our curious machine, soon your idea of nature will become immune to quantum weirdness. Our device, like a vaccination against Covid-19, will protect you from weirdness for your entire life. Only one injection is needed.

\subsection{What Gödel and Turing accomplished}

There is a stark contrast between the "boundary problem model" we are discussing, and what is usually considered a boundary problem for PDE's. A typical boundary condition problem would be to say that a PDE $\psi$ is defined over domain $\Omega$ and the boundary condition would be that $\psi=0$ everywhere on $\partial \Omega$. That information is decisive in determining the unique solution to the differential equation. We are adapting that technology for our own purposes, and we are turning the tables. Instead of using the boundary condition to determine which PDE solution is correct, we start with the differential equation solutions, and turn our attention to understanding the odd boundary conditions. 
Gödel's 1933 paper on the undecidability proposition is an example of what we are trying to do. He never discusses boundary conditions. He translated logical statements (like "arithmetic is complete and consistent") into a linear sum of prime numbers, then used those sums to prove an "incompleteness theorem," and an "inconsistency theorem." His labyrinthine and impenetrable argument demonstrated that no system of axioms is able to prove that it is complete and consistent. Gödel thereby demonstrated that David Hilbert's 1928 proposal to axiomatize the Principia Mathematica, and Cantor's set theory and all of arithmetic was impossible. $(37,40)$

We are also following in the footsteps of Turing's 1936 paper that proved that an algorithm to solve the halting problem ("Can we avoid an infinite loop?") for all computer programs cannot exist. (57)

The mathematical machines built by Gödel and Turing were convoluted and inscrutable and far more mazelike than the machinery we are building. What they proved is that mathematical statements cannot always be classified as true or false. There is a third choice: undecidable. A mathematical statement (like the Riemann hypothesis) could be true, false, or undecidable.

What do Gödel and Turing's mathematical machines have in common with the engine we are building? Both Gödel and Turing took simple, orderly, and well-defined technologies and built peculiar devices to solve a larger problem concerning mathematical axioms and logic. They demonstrated where the perimeter of mathematics was and proved that efforts to transcend that perimeter were doomed to failure. The perimeter of mathematics cannot include axioms and proofs that all of arithmetic is logical and consistent.

We will commandeer a different mathematical technology to attack a different problem. PDE equations and boundary problems provide a well-defined set of terminology, equations, expectations, and patterns of thought which we will use to build a game simulating reality. The purpose of our game will be to prove whether quantum weirdness can exist, and if not, why not? Our hypothesis is that quantum weirdness lies outside the perimeter of mathematics.

Since no definition of "quantum weirdness" exists, we will boil down that amorphous blob into a single dot.

The outcome of Gödel and Turing's work was to change the direction of the entire discipline of mathematics. Hilbert, in his 1928 book, Foundations of Mathematical Logic, had been leading mathematics toward imperialistic axiomatization. Hilbert was motivated by a desire to "put math back in the driver's seat" in control of all sciences. Gödel and Turing defeated that axiomatization campaign.

Yet it was not fruitless. The phoenix that rose out of those ashes was the invention of computers. Those historical battles gave birth to the Turing machine, and now this article is being written on a Turing machine instead of a typewriter.

\subsection{Rules of our game}

In this article, as we said, domain $\Omega$ is the region where equation $\mathbf{u}_{\mathbf{t t}}=\mathbf{c}^{\mathbf{2}} \mathbf{u}_{\mathbf{x x}}$ is defined. In one-dimension, there are two complex solutions, one travelling to the left $\left(\psi_{\mathrm{L}}=\boldsymbol{e}^{i(k x+\omega t)}\right)$, the other to the right $\left(\psi_{\mathrm{R}}=\boldsymbol{e}^{i(k x-\omega t)}\right)$. Although such a 2-way solution is typical of second order linear PDE's, our waves are off-center and not mirror images of each other. It is not enough to look to the equation $\mathbf{u}_{\mathbf{t t}}=\mathbf{c}^{2} \mathbf{u}_{\mathbf{x x}}$ for guidance. We are looking to the context within which $\mathbf{u}_{\mathbf{t t}}=\mathbf{c}^{\mathbf{2}} \mathbf{u}_{\mathbf{x x}}$ lives, to discover how the rules of our game should be written. We call our creation a "game," but we don't have a contest for you to play. By calling it a "game" want to engage your imagination and amusement. We are requesting you suspend your disbelief.

Our boundary condition at point $\boldsymbol{\alpha}$ spells out how these two solutions $\left(\psi_{\mathrm{L}}\right.$ and $\left.\psi_{\mathrm{R}}\right)$ differ from one another. The plane wave to the left $\left(\psi_{\mathrm{L}}\right)$ can be subclassified as two different waves, depending on whether it encounters a particle. We will name those $\psi_{\mathrm{L}}$ and $\psi_{\mathrm{G}}$ respectively, where the "G" subscript stands for "ghost," for we will name it a "ghost wave" if it does not encounter a particle. To keep our model as simple as possible, we will consider

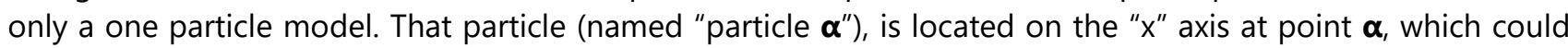
be stationary or moving. 
The reflected wave we define as moving to the right $\left(\psi_{R}\right)$ is noteworthy for several reasons. First, it is not just a mirror image of the same wave that was previously a plane wave moving left $\left(\psi_{\llcorner}\right)$. Why? Because as it makes a " $U$ " turn, $\psi_{\mathrm{R}}$ transforms into a Schrödinger wave that carries particle $\boldsymbol{\alpha}$ away to the right. Below we will provide the equations that define that metamorphosis.

\section{As you can see, there is a lot going on at point $\alpha_{\text {., }}$ which we are calling the "boundary condition" pinpointed at the $\partial \Omega$ boundary.}

Particle $\boldsymbol{\alpha}$ has two states it can be in. It may be visible to the incoming plane wave $\left(\psi_{\mathrm{L}}\right)$ in which case it will reflect that wave, so that wave $\psi_{\mathrm{L}}$ becomes $\psi_{\mathrm{R}}$. On the other hand, particle $\boldsymbol{\alpha}$ may choose (at random) to be transparent to the incoming plane wave $\left(\psi_{\mathrm{L}}\right)$, in which case the wave passes straight trough as if the particle didn't exist. In that case the leftward travelling wave can be called a "ghost wave" ( $\psi_{\mathrm{L}}$ becomes $\psi_{\mathrm{G}}$ ) still moving to the left. The reason it is a ghost is that our detectors can "see" particles but cannot see waves, so a wave carrying no particle is undetectable, like a ghost. We know of such waves only by inference.

Particle $\boldsymbol{\alpha}$ makes a random choice whether to be reflective or transparent vis-à-vis every incoming wave $\left(\psi_{\mathrm{L}}\right)$. There are many incoming waves. The particle has one and only one opportunity to become reflective. Its resting state or basic condition is transparent. That one time when it becomes reflective, it is instantly swept off its feet by the newly created Schrödinger wave moving toward the detector. The decisive boundary condition (i.e., the focus of this article) is that point $\boldsymbol{\alpha}$.

Point $\boldsymbol{\alpha}$ is the left boundary of domain $\Omega$, as we said. The detector is the right boundary, but it is not subject to such interesting rules.

You can see why we call this a "boundary condition problem" pinpointed at the $\partial \Omega$ border. But it is a knotty problem, orders of magnitude more convoluted than anything you will find in Wolfram Alpha.

Time is positive, so $(0<\mathrm{t})$ and time always moves in a positive direction, with no time reversal. The amplitude of the wave is bounded (because we will interpret it as a probability amplitude that is normalized):

$$
0 \leq\|\psi(x, t)\| \leq 1 .
$$

As noted above, we will also create a function $\boldsymbol{E}(\mathrm{x}, \mathrm{t})$. We define $\psi_{\mathrm{L}}$ and $\psi_{\mathrm{R}}$ and their interaction with particle $\boldsymbol{\alpha}$ to be a bi-directional wave which we will name $\boldsymbol{A}(x, t)$, recognizing that $\boldsymbol{A}$ is complicated and is defined on domain $\Omega$. We will formally define $\boldsymbol{A}$ below. The symbol $\boldsymbol{A}$ is the first letter of the ancient English and Viking alphabets.

Although this discussion of boundary conditions is unlike any you have ever seen before, you can easily learn the rules of how it works. When we discuss the double slit experiment below you will discover the advantage of this system, because the double slit problem is trivial to solve with our technology. No one else has ever solved it. Richard Feynman declared that the double slit experiment was the "central mystery of QM." He had no idea how to solve it, or even think about it.

\subsection{The central role of particle $\alpha$}

In our model the particle $\boldsymbol{\alpha}$ at the $\partial \Omega$ boundary plays a pivotal role, as we said. The particle makes a random choice whether to be reflective or transparent vis-à-vis each incoming $\psi\llcorner$ Lave.

Since particles are too small for us to see, what do we mean by the words reflective and transparent? We are describing how a particle affects a wave. If the wave changes direction after encountering the particle $\left(\psi_{\mathrm{L}}=\right.$ $\left.\boldsymbol{e}^{i(k x+\omega t)} \rightarrow \psi_{R}=\boldsymbol{e}^{i(x k-\omega t)}\right)$ then we label the particle reflective. But if the wave gives no evidence of encountering the particle, then we use the word transparent because the wave passed straight through unchanged. That $\psi_{\mathrm{G}}$ wave is like a ghost.

Mathematician John von Neumann asked a question that no one could answer. "The Schrödinger wave equation is deterministic, so how did randomness get into QM?" It is a fundamental and glaring question. TEW declares that randomness enters QM via the particle, which makes random decisions about whether to be reflective or 
transparent. Anyone who looks at particles under a microscope (albeit large particles if they are visible) and observes Brownian motion, can see that the nature of particles is to be erratic. (59)

Erwin Schrödinger did not believe in the existence of physical particles. He thought they could be replaced by wave packets. We disagree. His idea would obliterate the boundary that defines $\Omega$, and it would be unable to explain why $\psi_{\mathrm{L}}$ makes a " $\mathrm{U}$ " turn at particle $\boldsymbol{\alpha}$. $(33,38,54-55)$

\subsection{How to define "quantum weirdness"}

What do you know about "quantum weirdness"? We can only describe it: "Somehow things don't add up right!"(1,3,34-36,39,43,49-50)

Here is an example of quantum weirdness. David Mermin said, "QM has proved that the moon only exists when people look at it."

\section{Boundary conditions in a neutron interferometer experiment}

So far, we have been building a game at point $\boldsymbol{\alpha}$ on the $\partial \Omega$ boundary. Aside from the complicated interaction of waves impinging on and reflecting off particle $\boldsymbol{\alpha}$, the most remarkable aspect of our arrangement is the blue arrow representing a zero-energy wave starting at the detector, the arrow which we call an "elementary wave." Our game would be more interesting if it is modelled on reality, than if it simply a figment of our imagination.

In this section we will describe a neutron interferometer experiment, the goal of which is to illustrate how the blue arrow might behave if it were real. The experiment was published in published in 1992 in Physical Review $A$ by Kaiser, Clothier, Werner, et. al. $(42,60)$

Helmut Rauch in Vienna, Austria, established a research team that laid the foundations of neutron interferometry research. Their experimental work was well known and highly respected. They published many articles in leading journals of physics, including the article we are about to discuss.

Neutrons entered an interferometer where they bounced off various silicon blades. The blades divided the neutrons into two streams, $\psi_{1}$ and $\psi_{2}$, then the beams were recombined before they left the interferometer and went to a detector (Fig. 5). Where the streams divided there was an oscillating aluminum plate that changed the phase of $\psi_{2}$ compared to the phase of $\psi_{1}$, so the detector saw an interference pattern (a sine wave). They put in the upper beam $\left(\psi_{2}\right)$ a sample of bismuth of varying thickness. Bismuth slows down neutrons and neutron waves. When they increased the thickness of bismuth the amount of interference diminished, to the point that at $12 \mathrm{~mm}$ or more, all interference was gone. They reasoned that the upper wave packet $\left(\psi_{2}\right)$ had been so delayed that the lower wave packet $\left(\psi_{1}\right)$ had already left the interferometer before the upper wave packet arrived at the reunion point. That would be why interference diminished to zero.

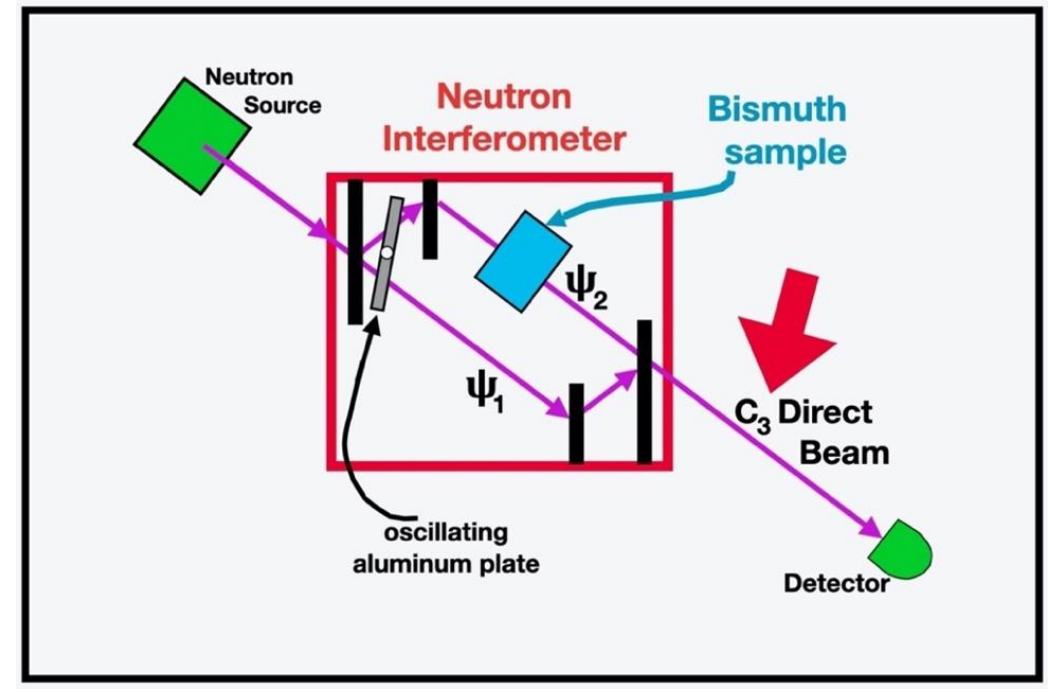

Fig. 5. Domain $\Omega$ extends from the neutron source to the detector. Kaiser et.al. sent a beam of neutrons into an interferometer, where silicon blades (in black) divided it into $\psi_{1}$ and $\psi_{2}$ which acquired an oscillating phase 
shift relative to one another. The $\Psi_{2}$ beam passed through a bismuth sample that slowed it down. After the beams were recombined, the height of sinusoidal waves diminished as more and more bismuth was added, eventually diminishing to zero interference.

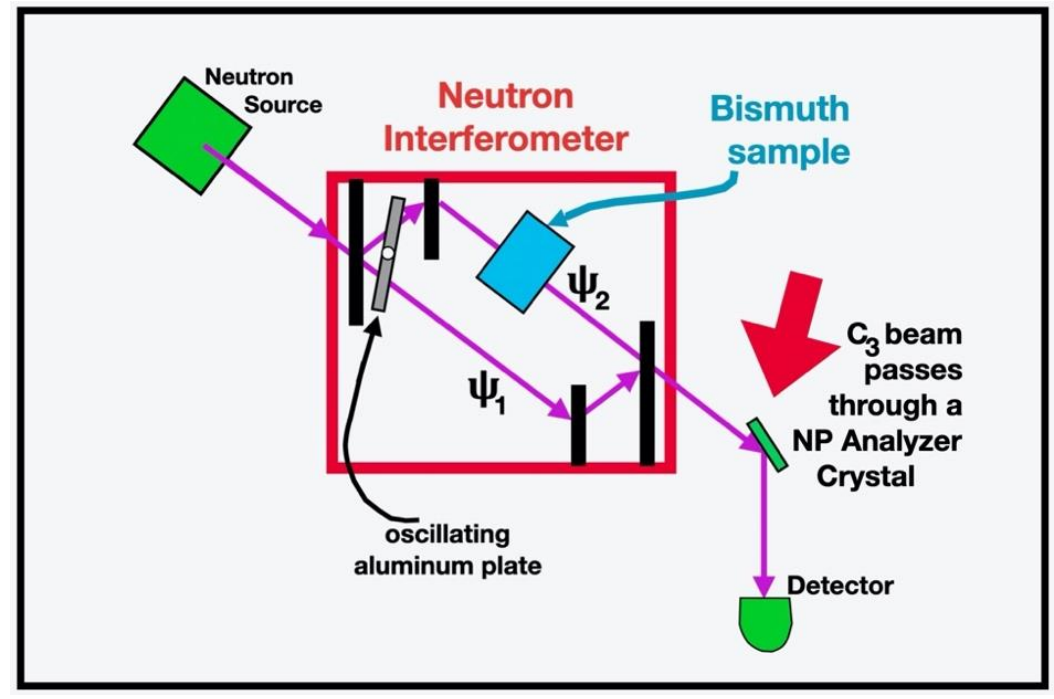

Fig. 6. An analyzer crystal is inserted in the exit beam of the interferometer.

They then repeated the same experiment with one tiny modification. They inserted a nearly perfect (NP) silicon crystal in the exit stream (Fig. 6), downstream from the interferometer, before the neutrons reached the detector. The crystal focused the beam, decreasing the spread of neutron wavelengths and increasing the height of the center of the Gaussian. Since that crystal was inserted downstream from the interference, it was expected to have no impact on the interference upstream inside the interferometer (Fig. 6).

To their astonishment (Table 1 and Fig. 7) the NP analyzer crystal restored robust interference. The researchers said they could not explain these data, and they said that QM could not explain these data.

\begin{tabular}{|c|l|l|}
\hline $\begin{array}{c}\text { Bismuth } \\
\text { width } \\
\text { in } \mathbf{~ m m}\end{array}$ & $\begin{array}{l}\text { Amount of interference in the } \mathrm{C}_{3} \text { Direct } \\
\text { Beam } \\
\text { Without Analyzer Crystal }\end{array}$ & $\begin{array}{l}\text { Amount of interference in the } \mathrm{C}_{3} \text { Beam } \\
\text { With } \\
\text { NP Analyzer Crystal }\end{array}$ \\
\hline $\mathbf{0} \mathrm{mm}$ & $100 \%$ & $100 \%$ \\
\hline $4.01 \mathrm{~mm}$ & $\mathbf{5 7 . 3} \pm 1.0$ & $97.1 \pm 5.1$ \\
\hline $12.26 \mathrm{~mm}$ & $8.0 \pm 0.8$ & $89.6 \pm 4.4$ \\
\hline $16.15 \mathrm{~mm}$ & $1.8 \pm 0.8$ & $86.0 \pm 4.8$ \\
\hline $20.08 \mathrm{~mm}$ & $2.9 \pm 0.6$ & $95.2 \pm 5.2$ \\
\hline
\end{tabular}

Table 1. Height of the sinusoidal curve. (Data are copied from the righthand column of Tables III and VI, of the Kaiser, et. al. 1992 article.) 


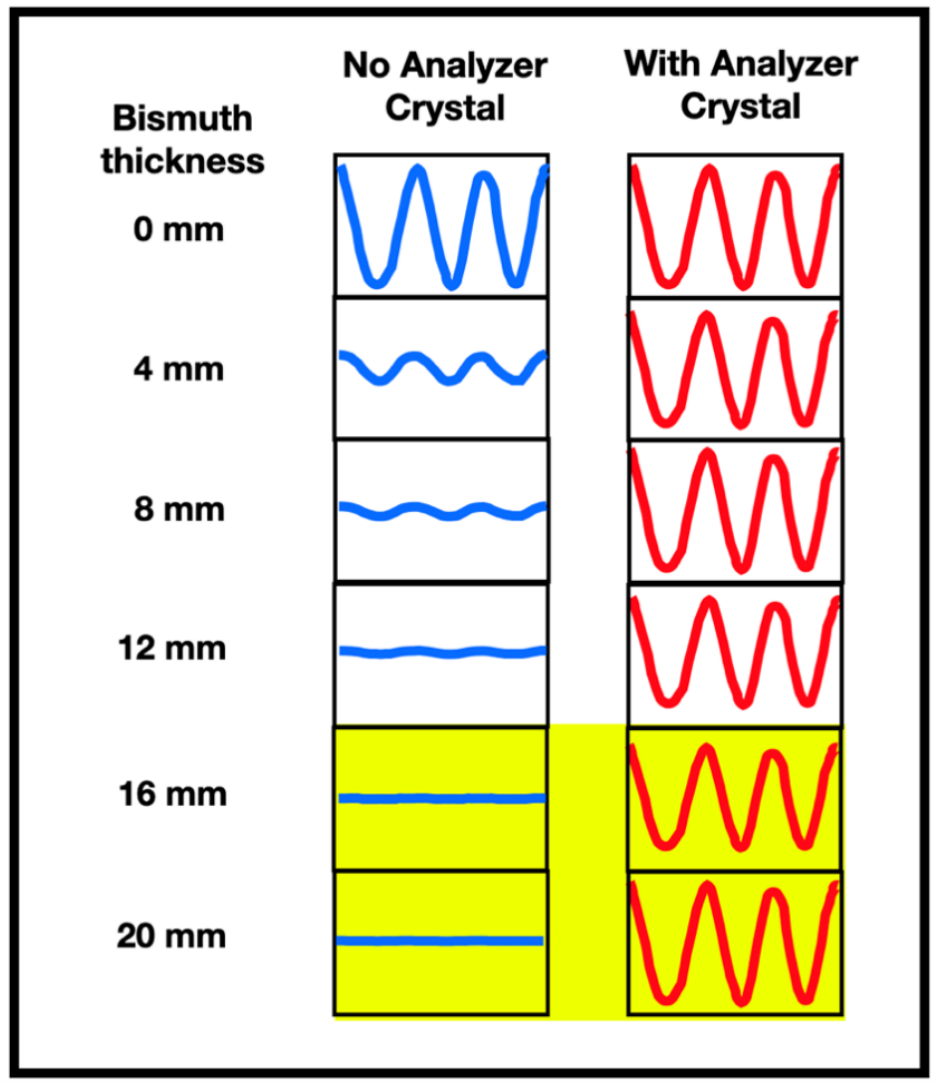

Fig. 7. Comparison of the interferograms with no NP analyzer crystal, compared to the presence of an NP analyzer crystal. In the yellow area, with 16 or $20 \mathrm{~mm}$ of bismuth, all interference died out (center yellow area) until an analyzer crystal was inserted, whereupon robust interference was restored (lower right yellow area). These curves come from the top and bottom graphs of Fig. 9, page 40 of the Kaiser, et. al. 1992 article.

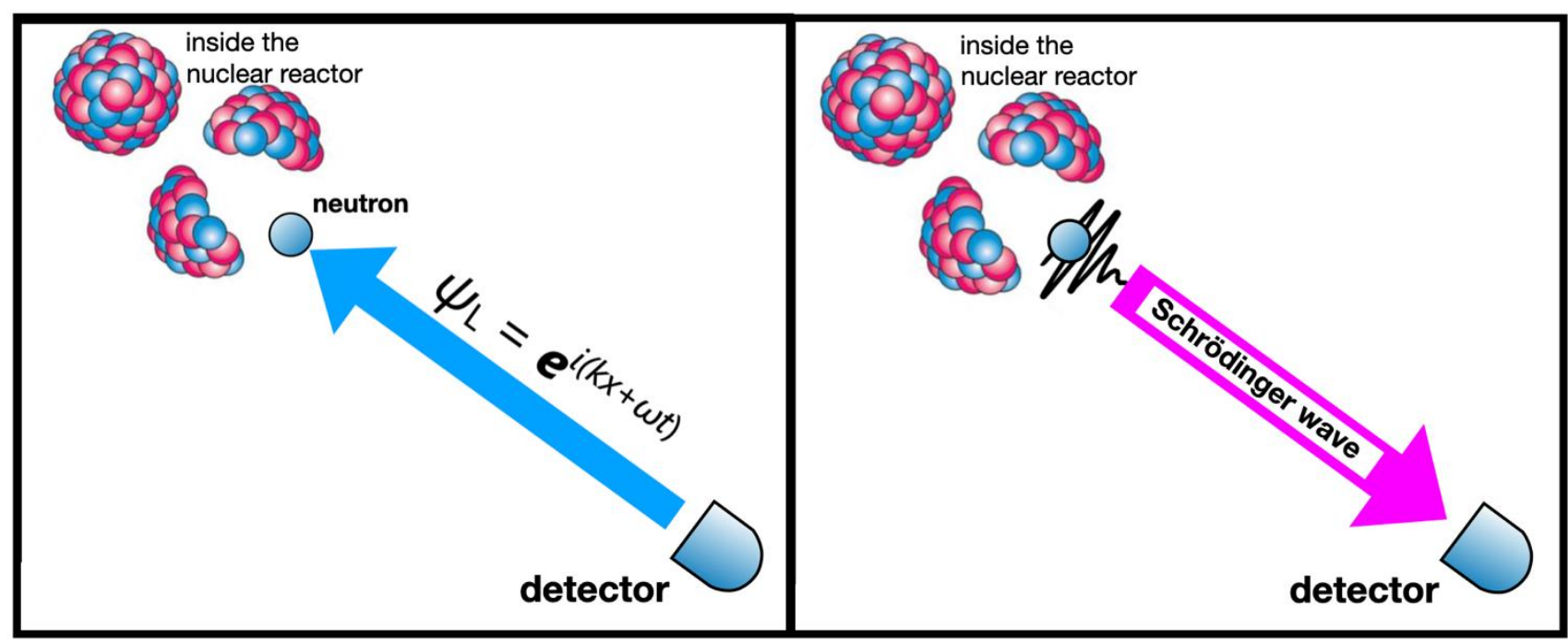

Fig. 8. Domain $\Omega$ extends from the nuclear reactor to the detector. Inside the reactor an atomic nucleus (red $\&$ blue composite upper left), as it splits, releases a neutron (blue sphere). A plane wave (blue) reflects off that neutron which is located at the $\partial \Omega$ boundary. As the wave reflects it become a Schrödinger wave, carrying the neutron to the detector.

The authors (Kaiser, et.al.) said they could not explain why an NP Analyzer Crystal placed downstream from the interferometer would produce such robust sinusoidal curves in the data. We say that if the presence or absence 
of an analyzer crystal causes or prevents wave interference, then the analyzer crystal must be upstream from the interference. This means waves are travelling in the opposite direction as the neutrons.

\subsection{Summary of the neutron interferometer data}

Let's review what we know from the Kaiser neutron interferometer data. There are two columns of data (Table 1 and Fig. 7). The middle column shows that the oscillating aluminum plate affects the neutron wave packets before the bismuth does, which could only happen if there were Schrödinger waves moving from the neutron source to the detector. The right-hand column of data shows that an analyzer crystal restores robust interference inside the interferometer, which could only happen if there were plane waves (elementary waves) moving from the detector up into the neutron source. Therefore, the data demonstrates that waves must be travelling in both directions, as we pictured in Fig. 8.

This experimental data show that the plane wave (elementary wave, $\left.\psi_{\mathrm{L}}\right)$ and the Schrödinger wave $\left(\psi_{\mathrm{R}}\right)$ co-exist, moving in opposite directions (Fig. 8).

In summary, in this and in many other experiments the blue arrow (called an "elementary wave") travels from the detector to the particle source, and a quantum particle follows that arrow backwards to the detector.

\section{The PDE wave equations defined on domain $\Omega$}

We have introduced the domain $\Omega$, which is where the PDE wave equation (Eq. 1) is defined. We have emphasized the complicated boundary conditions around particle $\boldsymbol{\alpha}$ which sits on and defines the $\partial \Omega$ boundary. That particle is like a force of nature when it changes from transparent to reflective and back to transparent. In this section we will derive the partial differential equations that transformation occurs.

We are about to do something astounding. We are going to give you 28 simple equations, each of which can be easily understood, and when you assemble them all into the machinery that we call the $\boldsymbol{A}$ model, you will discover that this machinery is able to describe the quantum world without any weirdness. The reason we can accomplish this is because we have adopted unique boundary conditions at particle $\boldsymbol{\alpha}$.

\subsection{Deriving our complex wave equations in one dimension}

To derive a one-dimensional equation for our plane wave travelling to the left, we begin with a standard Laplacian wave equation: $\mathbf{u}_{\mathbf{t t}}=\mathbf{c}^{\mathbf{2}} \mathbf{u}_{\mathbf{x x}}$ (Eq. 1).

We now consider a possible solution of Eq. 1:

$$
\begin{array}{r}
u=e^{i(k x \pm \omega t)}= \\
\cos (k x \pm \omega t)+i \sin (k x \pm \omega t)
\end{array}
$$

then

$$
\begin{array}{r}
\frac{\partial^{2} u}{\partial^{2} x}=k^{2} e^{i(k x \pm \omega t) \quad \text { and }} \\
\frac{\partial^{2} u}{\partial^{2} t}=\omega^{2} e^{i(k x \pm \omega t)} .
\end{array}
$$

Therefore

$$
\frac{\partial^{2} u}{\partial^{2} t}=c^{2} \frac{\partial^{2} u}{\partial^{2} x}=\omega^{2} e^{i(k x \pm \omega t)}=c^{2} k^{2} e^{i(k x \pm \omega t)} .
$$

This is true iff

$$
\omega^{2}=c^{2} k^{2} .
$$

Based on standard definitions of $\lambda$ (wavelength), $f$ (frequency), $\omega$ (angular frequency) and $k$ (wave number), we can say that 


$$
\text { velocity }=c=\lambda f=\frac{\omega}{k} \text {. }
$$

Therefore Eq. 7 is true. Therefore

$$
u=e^{i(k x \pm \omega t)}
$$

(which is also Eq. 2) is a solution of the wave equation (Eq. 1). If the $\pm \omega t$ is a minus sign, then the wave is moving to the right, which is the focus of section 2.2 below. If it is a plus sign then the wave is moving to the left, which we now turn to. Such a 2-way wave solution is well known, but, as we said, the waves to the left and right are dissimilar from one another.

\subsection{Plane wave moving to the left}

$$
\begin{gathered}
\text { We define } \\
\psi_{L}(x, t)=e^{i(k x+\omega t)}
\end{gathered}
$$

as our complex plane wave moving to the left. Note that it carries no operator for energy or momentum. It is just a garden-variety plane wave.

According to TEW most elementary waves are this kind of wave. It is a wave that is ghost-like because we have no way of detecting such a wave. In any volume of space there are an infinite number of waves like this, but only a finite number of particles. Our detectors can "see" particles but not waves. The only way we know that this vast population of particle-less waves exist is by inference.

Eq. 10 cannot be developed into a time dependent Schrödinger equation (TDSE). If you try to do so you will find it is impossible because of the " + " sign in the exponent becomes a minus sign (" $-")$ intruding into your would-be-Schrödinger equation. Therefore, Eq. 10 cannot be developed into a TDSE the way we are about to develop Eq. 11 into a TDSE.

This means that the character of a plane wave changes when it reflects off a particle because in the exponent the "+" sign in the exponent becomes a minus sign ("-"). That reversal of directions allows the wave to develop Schrödinger capabilities. Reflecting off a barrier is a toggle switch that changes an innocuous plane wave into an impressive Schrödinger wave.

\subsection{Waves moving to the right}

If a plane wave travelling to the left reflects then it will become a mirror image of itself, moving to the right. $\psi_{\mathrm{R}}$ is our name for a wave moving to the Right:

$$
\psi_{R}(x, t)=e^{i(k x-\omega t)}
$$

This wave might deceive you into thinking it is a garden-variety plane wave. But no. It is instantly transformed into a Schrödinger wave as follows.

\subsection{The TISE}

The particle will only become reflective and interact with a wave if the de Broglie wavelength is the same, in other words if

$$
\lambda=\frac{2 \pi \hbar}{m v}
$$

Where $m$ and $v$ are the mass and velocity of the particle. We likewise define

$$
k=p / \hbar \text { and } p=m v
$$

Where $p$ is the momentum of the particle.

We define 


$$
\begin{gathered}
E=\text { kinetic energy }+ \text { potential energy } \\
E=\frac{1}{2} m v^{2}+u=\frac{p^{2}}{2 m}+u
\end{gathered}
$$

Taking the second derivative $\partial^{2} / \partial x^{2}$ of the wave function $\Psi_{R}=e^{i(k x-\omega t)}$ (Eq. 11) we get:

$$
\begin{aligned}
& \frac{\partial^{2} \psi_{R}}{\partial x^{2}}=\frac{\partial^{2}}{\partial x^{2}}\left(e^{i(k x-\omega t)}\right)=(i k)^{2} \psi_{R}=-k^{2} \psi_{R}=\frac{p^{2}}{\hbar^{2}} \psi_{R} \\
& \hbar^{2} \frac{\partial^{2} \psi_{R}}{\partial x^{2}}=p^{2} \psi_{R}
\end{aligned}
$$

Multiplying both sides of Eq. 15, $\left[E=\left(p^{2} / 2 m\right)+u\right]$, by $\psi_{R}$, we get:

$$
E \psi_{R}=\frac{p^{2} \psi_{R}}{2 m}+u \psi_{R}
$$

and inserting Eq. 17 we get the Time Independent Schrödinger Equation (TISE):

$$
E \psi_{R}=-\frac{\hbar^{2}}{2 m} \frac{\partial^{2} \psi_{R}}{\partial x^{2}}+u \psi_{R}=\mathbf{T I S E}
$$

\subsection{The TDSE}

The time dependent equation can be easily derived by differentiating our wave equation

$$
\begin{gathered}
\psi_{R}=e^{i(k x-\omega t) \quad \text { by } \partial / \partial t:} \\
\frac{\partial \psi_{R}}{\partial t}=-i \omega \psi_{R}
\end{gathered}
$$

We define $E=\hbar \omega$. Multiplying that by $\psi_{R}$ we get:

$$
\begin{array}{r}
E \psi_{R}=\hbar \omega \psi_{R} \\
-\frac{i}{\hbar} E \psi_{R}=-i \omega \psi_{R}=\frac{\partial \psi_{R}}{\partial t} \\
E \psi_{R}=-\frac{\hbar}{i} \frac{\partial \psi_{R}}{\partial t}=i \hbar \frac{\partial \psi_{R}}{\partial t}
\end{array}
$$

We can substitute that into the Eq. 19 (TISE) and that gives us:

$$
i \hbar \frac{\partial \psi_{R}}{\partial t}=-\frac{\hbar^{2}}{2 m} \frac{\partial^{2} \psi_{R}}{\partial x^{2}}+u \psi_{R}
$$

which is the Time Dependent Schrôdinger Equation, TDSE.

\subsection{Summary of these equations}

We have shown how one and only one plane wave $\psi_{\mathrm{L}}$, reflecting off a particle, reverses direction and becomes a Schrödinger wave $\psi_{\mathrm{R}}$ capable of carrying that particle away from its Source. This is remarkable. We have reduced the entire weirdness of QM into 25 simple equations, each of which makes sense, and quantum weirdness vanishes, as we are about to demonstrate.

\subsection{Definition of $\boldsymbol{A E}$}

We will now define an "elementary wave $\boldsymbol{A}$ " which is a convenient way to cluster several other variables such as $\psi_{\mathrm{L}}$ and $\psi_{\mathrm{R}}$ into a powerful variable $\boldsymbol{A}$ that will be the center of our discussions. $\boldsymbol{A}$ is also defined over domain $\Omega$. 
Since we are working in one dimension, we will define the wave function $\boldsymbol{E}$ for the specific circumstance we need, which is a one-dimensional wave living inside a very peculiar and restricted setting of domain $\Omega$. Our goal is not to produce a definition that is generalizable, but to define an unprecedented idea of what we mean by a "wave" in a manner that uses boundary conditions never previously imagined, because our wave reverses direction under certain boundary circumstances.

AE will be a wave with independent variables

$$
x \in \mathbb{R} \text { and } t \in \mathbb{R}
$$

that cannot stand still. It can only travel to the left or to the right. Our domain $\Omega$ is finite, so $(-\infty<x<+\infty)$. We are primarily interested in $\mathrm{x}$ in the range from point $\boldsymbol{\alpha}$, which is where the particle is located, to the detector. In our model the particle source is on the left and detectors are on the right. Time is positive, so $(0<\mathrm{t})$ and time always moves in a positive direction, with no time reversal. The amplitude of the wave is bounded (because we will interpret it as a probability amplitude that is normalized):

$$
0 \leq\|\psi(x, t)\| \leq 1 .
$$

We define

$$
\begin{aligned}
& \text { If it is moving to the left, } \\
& \text { where } \mathrm{A} \text { is a constant } \\
& \text { If it is moving to the right, } \\
& \boldsymbol{E}(\mathrm{x}, \mathrm{t}) \equiv \psi_{R}(\mathrm{x}, \mathrm{t})=\text { a Schrödinger wave }
\end{aligned}
$$

Every Schrödinger wave in our model travels to the right and was originally a plane wave moving to the left. Our model is only for free particles. In our definition a plane wave can only reflect and reverse directions if it encounters a particle that we classify as "reflective." A particle can only become "reflective" if the incoming wave has a de Broglie wavelength corresponding to the momentum of the particle: $\lambda=(h / p)$.

In our model there is a one-to-one relationship between Schrödinger waves and particles, the one becoming attached to the other. Nevertheless, for every particle there are an infinite number of plane waves $\left(\psi_{\mathrm{G}}=\boldsymbol{e}^{i(k x+\omega t)}\right.$ which never encounter a reflective particle. We say these waves "never see a particle." As we said, these are referred to as "ghost waves" which we won't speak of much.

\section{A double slit experiment}

The elementary wave game that we are presenting proves its value when it comes to understanding the double slit experiment. Feynman said that the double slit experiment embodies what he called the "central mystery of QM." He said that QM cannot explain it. In our model the double slit experiment is straightforward and simple (Fig. 9).

Since there is a lot going on in Fig. 9, let's start by getting oriented. In the top of Fig. 1 you saw a particle $\boldsymbol{\alpha}$ on the left, a detector on the right, and a blue arrow from the detector to the particle, representing plane wave $\psi_{\mathrm{L}}$. In Fig. 9 you see the same thing. Particle $\boldsymbol{\alpha}$ is on the left (inside the particle gun), the detector is on the right (it is called a "target screen) and several blue arrows going from the detector to the particle, representing plane waves. 


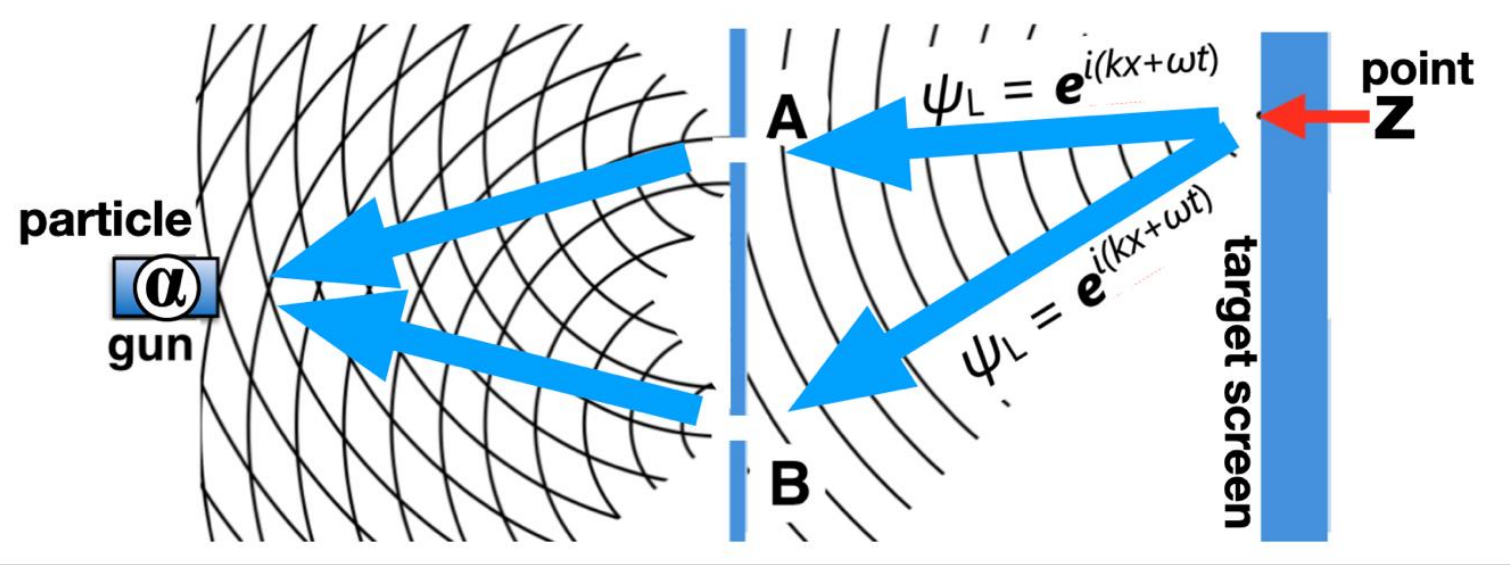

Fig. 9. Domain $\Omega$ extends from particle $\boldsymbol{\alpha}$ on the left to the target screen which is the "detector" on the right. Blue arrows representing plane waves $\psi_{\mathrm{L}}$ originate at the detector (at point $z$ ) and move to the left, impinging on particle $\boldsymbol{\alpha}$ (in the particle gun). There is wave interference caused by the two slits. This diagram implies that "point $z$ " is only one of a zillion points on the target screen doing the same thing.

Since these waves are inside a box with a double slit barrier in the middle, the plane waves move to the left in semicircles, penetrate the two slits as the waves travel to the left, and then there is wave interference. You notice "point $z$ " on the right side, which is the origin from which these waves are emanating. Why just one single point? The implication is that every single point on the target screen is putting out its own elementary waves. The waves coming out of " $z$ " don't mingle with the waves coming from other points. They are snobbish and unfriendly to waves from other points of origin.

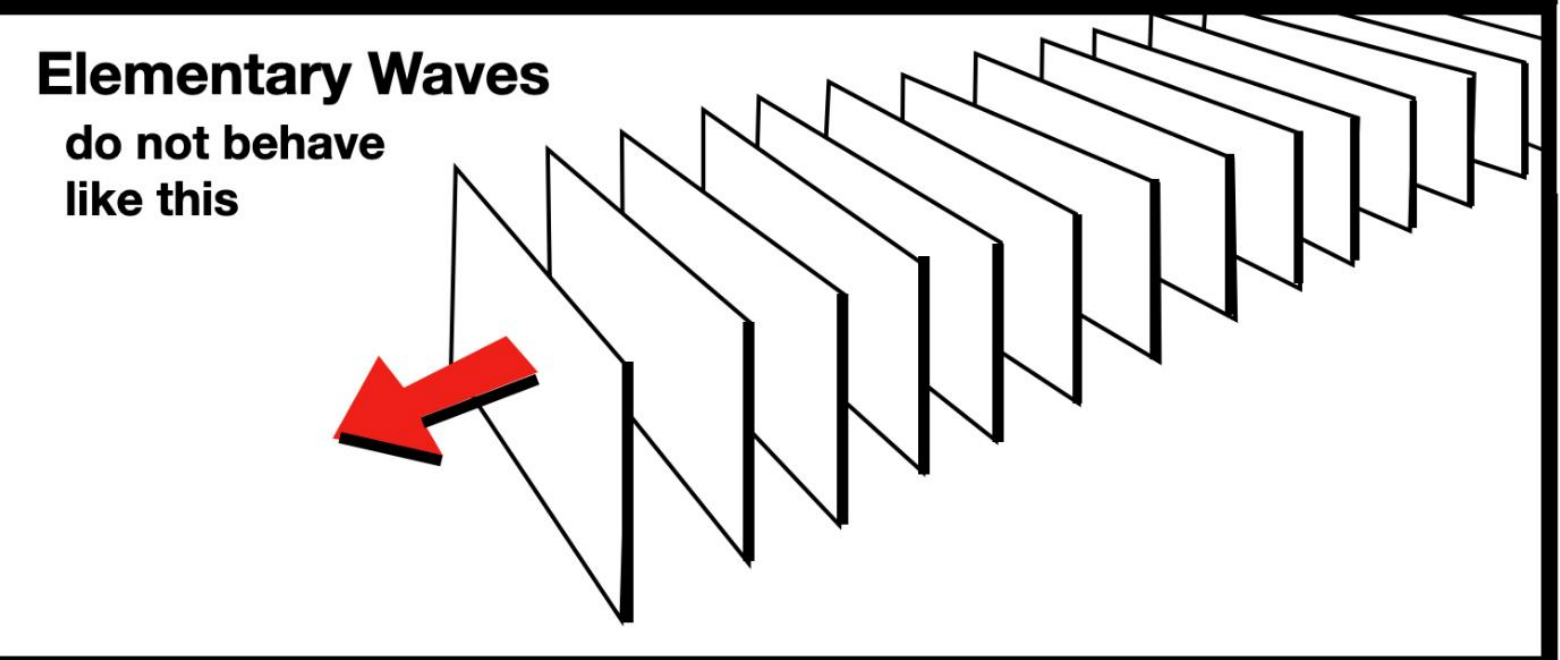

Fig. 10. Although the equation we use, $\left[\psi=e^{i(k x \pm \omega t)}\right]$, is called a "plane wave equation", nevertheless elementary waves do not form the parallel planes shown in this diagram (see text).

The term "superposition additivity" has more than one meaning in the world of elementary waves.

1. The wave equation $\left(u_{t t}=c^{2} u_{x x}\right)$ is linear and therefore different solutions can be superimposed and added in linear combinations. But that is only true for different solutions to an elementary wave equation that comes from one unique point on the target screen.

2. But a collection of elementary rays is like a collection of trajectories each with its unique wave equation. Rays arising from different points $\left(z_{1}, z_{2}, z_{3}, \ldots z_{n}\right)$ on the target screen, cannot be added together to form the "plane waves" shown in Fig. 11. This is because there is an assortment of wave equations, not necessarily related to one another, one arising from each point $\left(z_{1}, z_{2}, z_{3}, \ldots z_{n}\right)$. 
Returning to the case where all the elementary waves originate at the same point " $z$ " on the target screen, the $\psi_{\mathrm{L}}$ wave through slit A interferes with the $\psi_{\mathrm{L}}$ wave through slit B, which affects the amplitude with which the $\psi_{\mathrm{L}}$ wave from point " $\mathrm{z}$ " arrives at particle $\boldsymbol{\alpha}$. As we mentioned, it is not just point $z$. Every point on the target screen is sending waves to compete for the attention of particle $\boldsymbol{\alpha}$. That particle can only respond to one of them, and it must be one with a wavelength corresponding to the de Broglie wavelength of the particle. It is a random decision that is influenced by the amplitude of the incoming wave, which is determined by the wave interference of which we just spoke.

When we say the "particle can only respond to one of them," we mean that the particle turns from transparent to reflective as one wave $\psi_{\mathrm{L}}$ approaches but remains transparent for all other approaching waves. That lucky wave $\psi_{\mathrm{L}}$ that randomly won the lottery is reflected by the mirror (which is what particle $\boldsymbol{\alpha}$ looks like), and therefore $\left(\psi_{\mathrm{L}}=\boldsymbol{e}^{i(k x+\omega t)} \rightarrow \psi_{\mathrm{R}}=\boldsymbol{e}^{i(x k-\omega t)}\right)$ becomes $\psi_{\mathrm{R}}$ which instantly transforms into a Schrödinger wave that picks up particle $\boldsymbol{\alpha}$ and carries it out of the gun, heading for home (i.e., point $z$ ), using one and only one of the two slits (it doesn't matter which slit).

When the Schrödinger wave $\psi_{\mathrm{R}}$ emerges from point $\boldsymbol{\alpha}$ carrying particle $\boldsymbol{\alpha}$, that constitutes wave function collapse. Therefore, the double slit experiment is deterministic as soon as particle emission occurs. The particle is not subject to any further wave interference. Its destiny is to make a dot at point " $\mathrm{z}$ " on the target screen. It pursues that destiny with a probability of one.

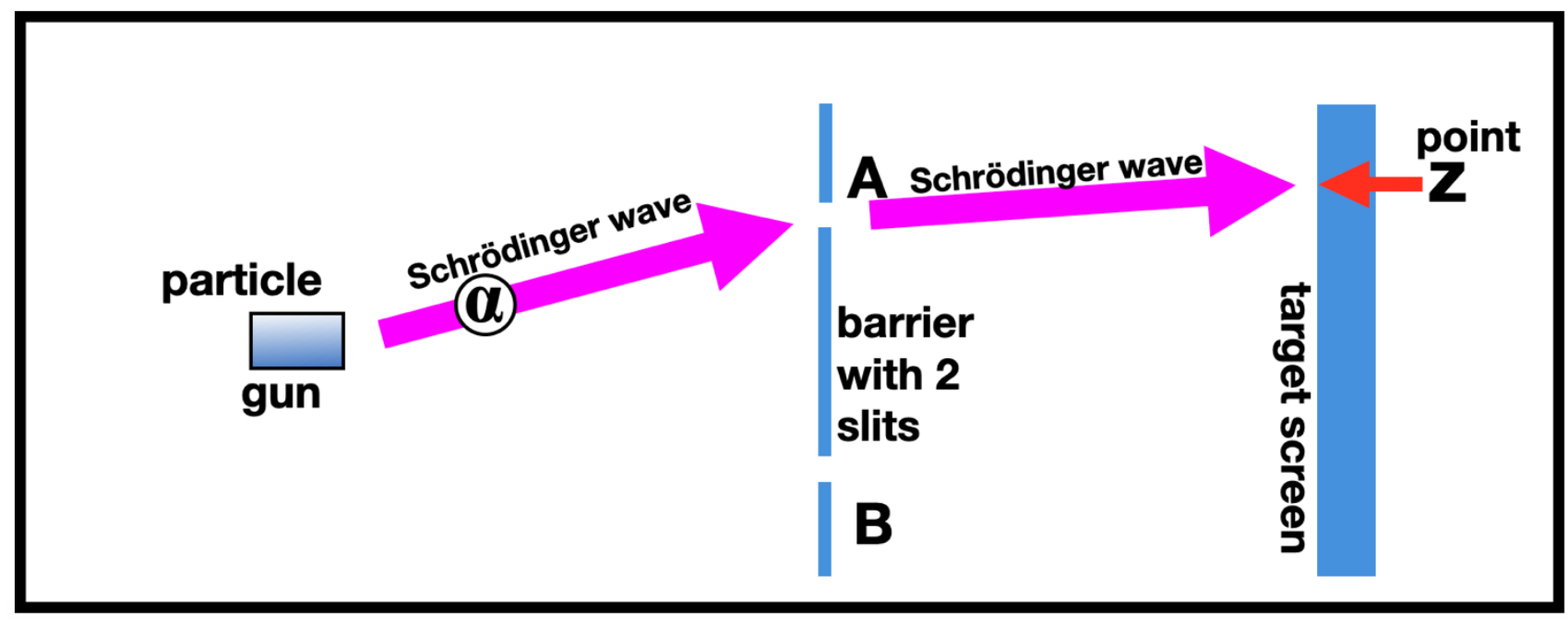

Fig. 11. In the double slit experiment the particle $\boldsymbol{\alpha}$ reflected only one of the zillions of impinging waves in Fig. 9. Upon reflection, $\psi_{\mathrm{L}}$ becomes $\psi_{\mathrm{R}}$ which instantly becomes a Schrödinger wave carrying particle $\boldsymbol{\alpha}$ away from the gun. With a probability of one it will strike point "z." It is following backward the trajectory of $\psi_{\mathrm{L}}$.

As you can see in Fig. 10, the Schrödinger wave has a predetermined trajectory that will lead inevitably to making a dot at point " $z$ " on the target screen. We can say three things about that trajectory: it is tracing backwards the path of least action that the winning $\psi_{\mathrm{L}}$ wave had travelled from point " $\mathrm{z}$ " to the particle gun. The second thing that we know is that our knowledge of that trajectory is limited by the Heisenberg uncertainty principle, which balances $\Delta \mathrm{x}$ against $\Delta \mathrm{p}$. The third thing to say is that the particle goes through one and only one of the two slits, and it doesn't matter which slit.

It is easy to show that this mechanism will produce the expected interference fringe pattern (wave pattern) on the target screen. Eq. 34 and Fig. 12 explain why. They depict the interference wave pattern on the target screen as the same, without ever referencing whether the waves travel left to right or right to left. Let " $L$ " be the length of the upper trajectory starting at the particle gun, passing up through slit $A$, then continuing down to point " $\mathrm{z}$ " on the target screen. Let " $\mathrm{M}$ " be the length of the lower trajectory starting at the particle gun, passing down through slit $B$, then continuing across to point " $z$ " on the target screen. Let " $\lambda$ " be the wavelength. Then we find that: 


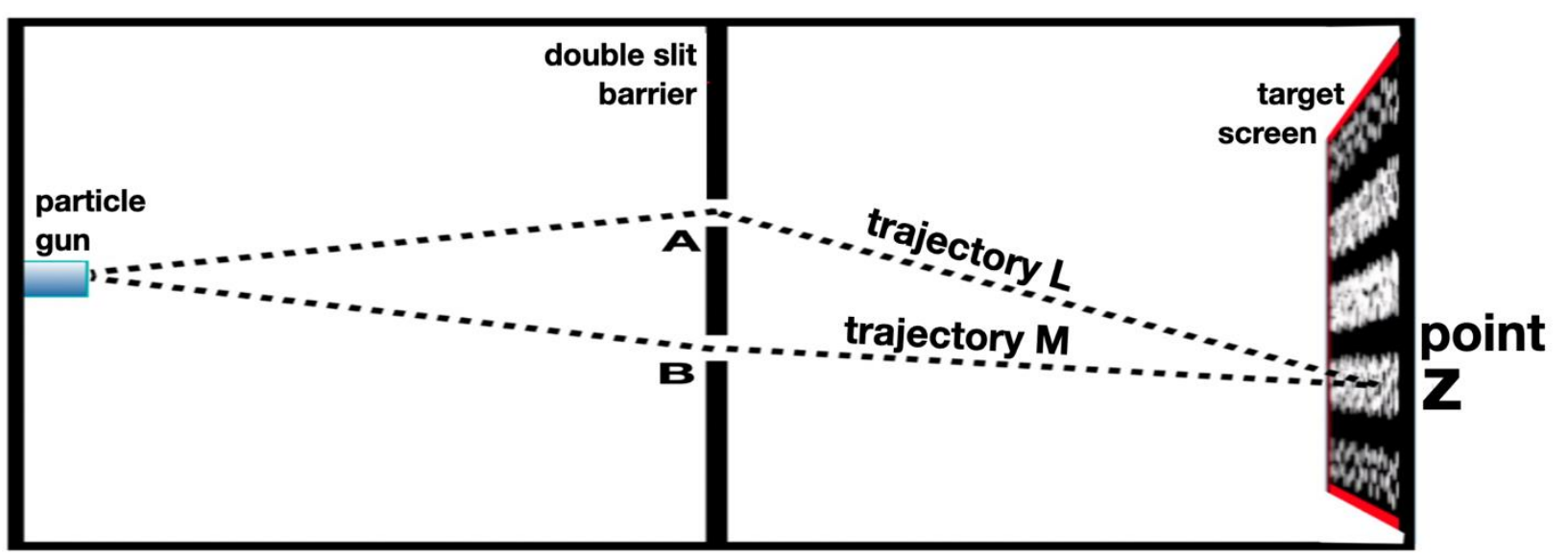

Fig. 12. This diagram compares the length of two trajectories (" $L$ " versus " $M$ ") between the gun and point "z." This diagram is the basis for Eq. 34. You get the same diagram and the same Eq. 34 if the waves are travelling left to right, or right to left. This is why TEW and QM produce identical patterns on the target screen.

$$
\text { Phase of the wave }=[(\text { length of } \mathbf{L}-\text { length of } \mathbf{M}) \text { modulo } \lambda] \times\left(\frac{2 \pi}{\lambda}\right)
$$

The phase of the wave, measured in radians, tells us the vertical position of the dot in the wave pattern on the target screen. The equation does not mention whether the waves are travelling right or left. In other words, the equation explains why the QM and TEW explanations produce identical patterns on the target screen.

\subsection{Complementarity}

"Complementarity" means that if we know which slit was used by the particle in a double slit experiment, then the wave pattern on the target screen vanishes. TEW explains this phenomenon without attributing it to human consciousness. To discover which slit, we must position a dim lamp inside the experimental apparatus, along with a detector, so we can detect a particle when it passes through one of the slits.

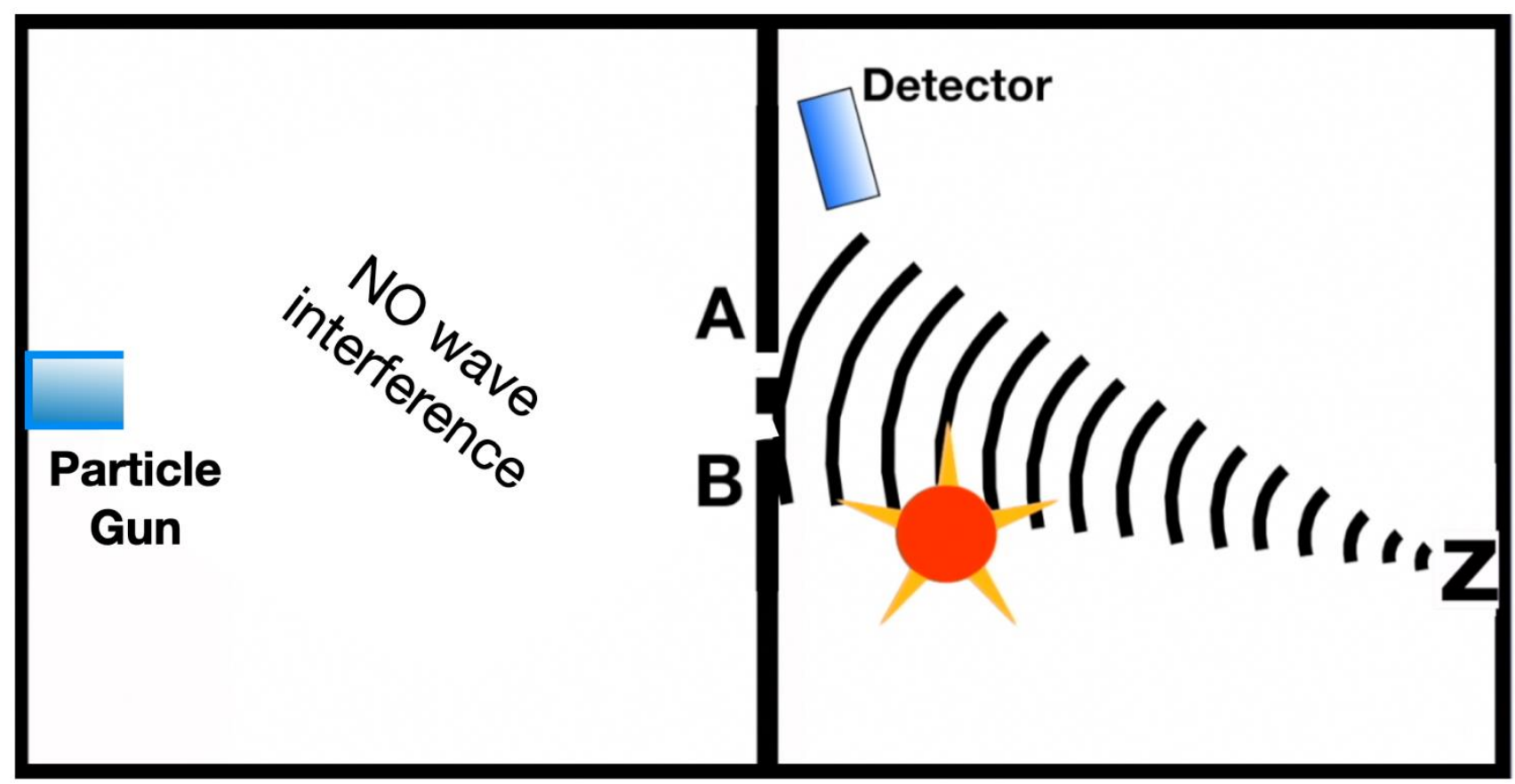

Fig. 13. To know which slit we put a lamp and detector inside the box. No matter how little energy of is used, it is still infinitely more energy than is carried in the zero-energy elementary waves coming from point $z$. That energy poisons the elementary waves. It destroys their memory of which point they were born at. 
The dim lamp emits infinitely more energy than the zero-energy elementary waves passing backwards through that slit. It poisons the elementary waves, so they lose their superposition additivity. As we said above, the term "superposition additivity" has more than one meaning. Superposition additivity of different solutions of a linear differential wave equation is intrinsic to the nature of linear differential equations. But when we speak of "superposition additivity" between elementary waves originating from different positions on the target screen $\left(z_{1}, z_{2}, z_{3}, \ldots z_{n}\right)$, there are many differential wave equations, one for each position $\left(z_{1}, z_{2}, z_{3}, \ldots z_{n}\right)$. Therefore, there is no superposition additivity of those neighboring waves, and therefore no "plane waves" as shown in Fig. 11.

If an elementary wave originates from point $z_{17}$ on the target screen, and if that wave happens to trigger a reflective particle $\boldsymbol{\alpha}$ to follow a Schrödinger wave, then that particle will make a dot at point $z_{17}$ and not at some other point on the target screen. Point $z_{17}$ is both the birthplace and the death-place of that elementary wave. Thus, each elementary wave is tethered or anchored to its place of birth, as if there were an elastic band attached from its birth location to the elementary wave throughout the wave's brief life.

However, a lamp inside the equipment will sever that anchor and destroy that tethering.

"Superposition additivity" among rays originating from neighboring points does not exist in the elementary wave world, because each neighboring trajectory has a different wave equation, as we said before. What is remarkable is that when elementary waves from point " $\mathrm{z}_{17}$ " penetrate both slits $A$ and $B$, they preserve their superposition additivity because they all come from the same origin (point " $\mathrm{z}_{17}$ ") and therefore from the same wave equation. However, when we insert a lamp and detector inside the double slit experiment, the lamp has infinitely more energy than the zero energy elementary waves, and it destroys their brotherhood.

Therefore, when the lamp is "on" the waves through slits A and B act as if they originated at points A and B, and each has a unique wave equation as they move toward the particle gun. As the waves impinge on the particle gun, they do not remember that they were born at point " $\mathrm{z}_{17}$ ". Their memory was erased by the energy of that lamp. What they are now thinking is "I was born at slit A" or "I was born at slit B." Since waves originating from different points do not possess superposition additivity in the elementary wave world, therefore there is no wave interference between the waves through slits $A$ and $B$ when the lamp is on.

What does the wave pattern on the target screen mean? The interference wave pattern on the target screen is a picture of the wave interference impinging on the particle gun. If, because of the presence of a lamp, there is no such interference of waves impinging on the gun (as we just said), then the target screen will report the truth: "No wave interference." This explains Complementarity.

\section{A proposed experiment}

In this section we will show you the design of a new experiment, never conducted, for which TEW and QM predict divergent outcomes. Fig. 21 shows the design of the experiment.

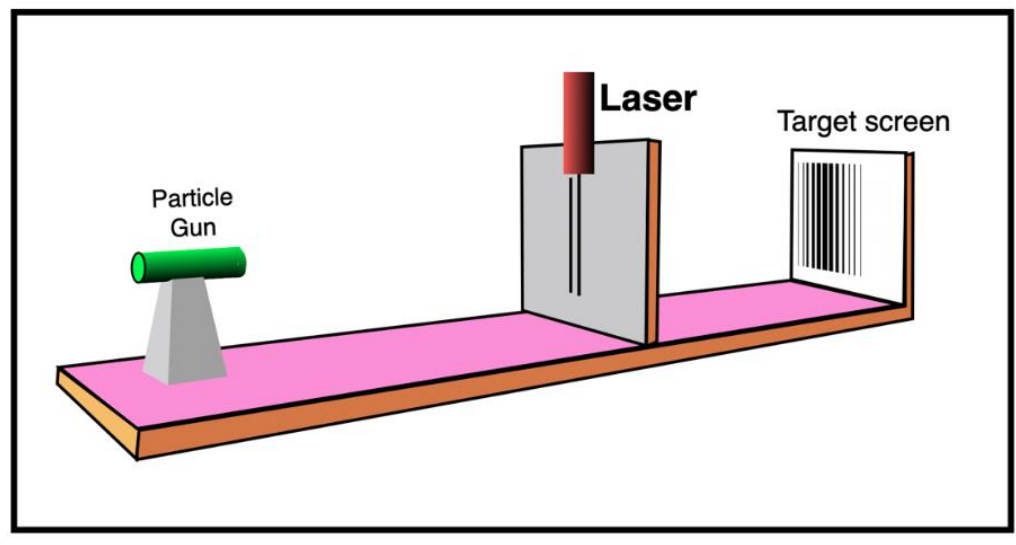

Fig. 21 If particles are fired one at a time, with a pause before the next particle, and if both slits are open until a particle is emitted by the gun, and then the vertical laser closes the right slit simultaneously as a particle is emitted, then QM predicts you would see no pattern, but TEW predicts a wave pattern as shown. 
For most experiments TEW and QM predict the same outcome data. But if you introduce a moving part, it is possible to design an experiment in which TEW and QM predict divergent outcomes. Fig. 21 is a double slit experiment with something added. The idea is that TEW says that all wave interference occurs before a particle is fired from the gun (the interference is in proximity to the gun), whereas QM says that no wave interference occurs after a particle is fired from the gun (interference is in proximity to the target screen), therefore we can divide time into two parts at the moment when the gun is fired, and all TEW wave interference would be finished, whereas no QM wave interference would have occurred.

The apparatus shown in Fig. 21 includes a powerful laser above the right slit. The laser would be used to close the right slit at that instant when a particle is fired from the gun, and to keep the right slit closed for a moment. QM predicts that this would be a single slit experiment. So, no interference fringe pattern would appear on the target screen. With TEW however, all the wave interference would be finished and that would be evident in the wave pattern the particle makes on the target screen. It would be only the left half of the interference fringe data. That is what is shown in Fig. 21. Namely a wave interference fringe pattern skewed to the left.

The reader is invited to build this apparatus and conduct this experiment. We know of no one who is planning to conduct this experiment.

\section{Schrödinger's cat}

Some people have never heard of the Schrödinger cat paradox. We will devote this paragraph to describing it. This is an imaginary experiment that should be avoided by cat lovers. A cat, a closed tube of cyanide gas and a hammer are put into a sealed box for an hour. At random a mechanism may release the hammer, so it falls and smashes the tube, so the cat suffers brain death from cyanide. At the end of an hour the experimenters open the lid and look, observing whether the cat is dead or alive. You might think that prior to that observation the cat was either dead or alive, but humans don't know which. But that is NOT how QM understands it. Since QM claims that wave function collapse occurs only when you observe something, the cat's fate (life or death) does not occur until someone opens the box and looks. Prior to that time the cat was neither dead nor alive but was in a "superposition" of both states at the same time. When people looked, that is when the superposition collapsed and only at that moment did the cat become dead or alive.

This is hard to follow because it is so preposterous.

The Schrödinger cat paradox focuses on when wave function collapse occurs. "Wave function collapse" means that a decision is made. Of all the possible outcomes (of all the possible eigenstates) one is chosen to become reality. Because QM does not have elementary waves, therefore QM is forced to say that wave function collapse occurs when you measure or observe something.

In TEW wave function collapse occurs before a measurement or observation is made. Wave function collapse occurs at the $\partial \Omega$ boundary and is controlled by particle $\boldsymbol{\alpha}$. When we discuss Schrödinger's cat, it is not hard to decide where the $\partial \Omega$ boundary is located, namely it is the glass tube of cyanide gas which, if shattered, will cause the cat to suffer brain death.

\subsection{The meaning of "wave function collapse"}

"Wave function collapse" means there are many possibilities for what the future will be, but a choice must be made, and only one of those possibilities will become reality. For example, as a young person you might flirt with and date many people, but then you marry one. After that your future is different because all the other possibilities are gone. (2)

QM says that "wave function collapse" occurs when you observe or measure something. TEW says that wave function collapse occurs before you observe or measure something. Usually, it occurs at the particle gun when a particle is emitted following only one of the possible trajectories.

\subsection{Schrödinger's cat}

In the elementary wave machinery we are discussing, wave function collapse occurs when a particle leaves the gun. 
That is different than the world described by QM, where wave function collapse occurs when a measurement or observation is made. This difference of viewpoints impacts how Schrödinger's cat is understood.

In the elementary wave game it is obvious when wave function collapse occurs in the Schrödinger's cat scenario. There is a superposition of states if the hammer hasn't fallen because the future is undecided, and it could go either way. If the hammer falls and the cat experiences brain death, that is when wave function collapse occurs. Why? Because that is when a decision is made. Afterwards, if the cat is dead, it is permanent.

What do we mean by a "superposition of states"? We mean that the cat is completely alive and completely dead in the future, depending on the random chance of what happens with that hammer. There is nothing spooky or unusual about this. When this author drives his car at a time when he is exhausted and drowsy, his future is fully dead AND fully alive AND fully alive with brain damage, depending on whether he falls asleep at the wheel. Knowing that provokes the author's anxiety and anxiety keeps him alert.

We have now completed our comments on the elementary wave game. It turns out that there is second game that we will now introduce.

\section{Another mathematical game}

To discuss the Bell test experiments, we will now define a new game (called "Advanced Elementary Wave game"),

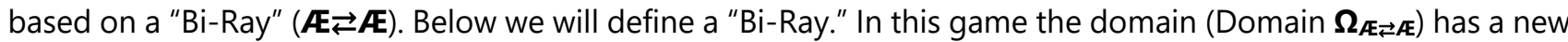
boundary condition. This is a one-dimensional domain that stretches from detectors named "Alice" on the left, to "Bob" on the right. The boundary condition for $\partial \Omega_{\mathbb{E} \rightleftarrows \mathbb{E} \text { is }}$ that the probability of a photon following a Bi-Ray is the amplitude of it following one ray times the amplitude of it following the countervailing ray. That condition at $\partial \Omega_{\mathbb{A} \rightleftarrows \mathbb{E}}$ boundary is the only assumption we need to account for the Bell test experimental results. This is an unusually powerful definition, because it is not only true at the boundaries ( $\partial \Omega_{\boldsymbol{E A Z A E}}$ ), but across the entire fiberoptic cable, which means across the entire domain $\boldsymbol{\Omega}_{\boldsymbol{E} \in \boldsymbol{E}}$.

This boundary condition game will explain the Bell test experiments in a way that is neither Einstein's nor QM's. Our model is closer to QM than to Einstein. We will develop a TEW model based on the Clauser, Horne, Shimony and Holt design of a Bell test experiments. $(1-4,32,58)$

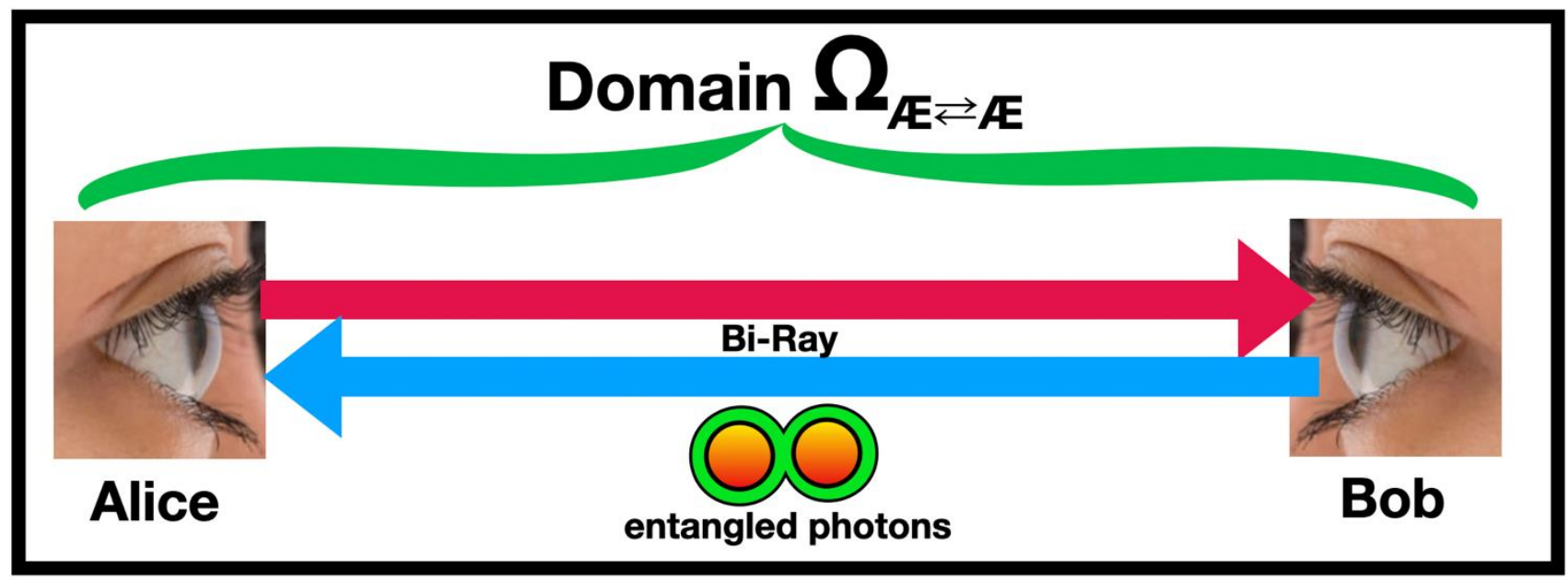

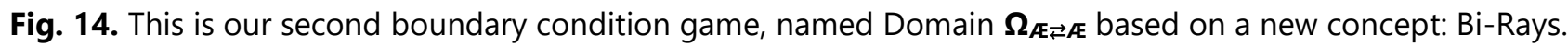

Now we need to define what we mean by the Bi-Ray. We postulate that we live in a universe in which there are, at every point, an infinite number of zero-energy elementary waves travelling in all directions and at all frequencies. That postulate means that every elementary ray has a mate, namely an identical elementary ray

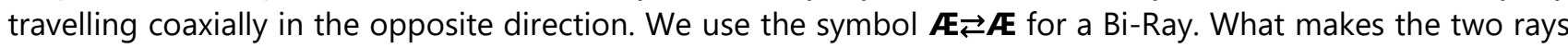
coherent is the photon(s) following that Bi-Ray. 
Our focus will be on the polarization of the $\boldsymbol{A}$ wave travelling to the left, versus the polarization of the $\boldsymbol{A}$ wave travelling to the right. We will focus on polarization and ignore the distinction we previously made between a plane wave and a Schrödinger wave.

Now we will discuss the elegance of our single boundary condition at $\partial \Omega_{\boldsymbol{A E} \rightleftarrows \boldsymbol{E} \text {. }}$ Given that single requirement, we can predict that the coincidence rate of the Bell test experiments will be $\boldsymbol{P}=\cos ^{2}\left(\boldsymbol{\varphi}_{2}-\boldsymbol{\varphi}_{1}\right)$ or $\boldsymbol{P}=\sin ^{2}\left(\boldsymbol{\varphi}_{2}-\right.$ $\left.\boldsymbol{\varphi}_{1}\right)$, where " $P$ " means "probability" and $\boldsymbol{\varphi}_{1}$ and $\boldsymbol{\varphi}_{\mathbf{2}}$ are the random angles of Alice and Bob's polarizers.

The difference between cosine and sine is that it depends on what technology is used to generate two entangled photons. For example, in Alain Aspect's experiment he used a calcium cascade source to generate 2 photons with the same polarization, and therefore the coincidence rate he discovered was $P=\cos ^{2}\left(\varphi_{2}-\varphi_{1}\right)$. If Aspect had used a different source (for example, one involving a Wollaston prism) that generated photons orthogonal to one another at birth, then his coincidence rate would have been $P=\sin ^{2}\left(\varphi_{2}-\varphi_{1}\right)$.

\subsection{Brief summary of the TEW results}

Some readers ask whether the TEW model of the Bell test experiments is "local" or "nonlocal." Beware that those words are charged with contentious meaning. Some scholars declare that TEW is a "nonlocal" model. Other scholars declare passionately that TEW is a "local" model. It is best to avoid the terms "local" and "nonlocal," because those terms are obsolete relics of the 50 year war between QM and Einstein's local realism vis-à-vis the Bell test experiments. Everyone can agree on how the TEW model works iff the words "local/nonlocal" are avoided.

For a century after Isaac Newton, people said that gravity was "nonlocal." The sun magically reached across empty space and interacted gravitationally with each planet. This "nonlocality" troubled Newton, who thought it was a weakness in his theory. Subsequently science developed the idea of a field. Each position in space was locally affected by a gravitational field.

Similarly, QM proposes that Alice's equipment magically reaches across empty space and sends a "nonlocal" signal to Bob's equipment faster than the speed of light. TEW solves that problem of the distance between Alice and Bob, without exceeding lightspeed. TEW defines a field all the way from Alice, through the 2-photon source, to Bob. Everywhere in that field (i.e., everywhere in the fiberoptic cable) the coincidence rate is $\cos ^{2}\left(\varphi_{2}-\varphi_{1}\right)$, as you will see.

The probability field is always the same intensity, $\mathrm{P}=\cos ^{2}\left(\varphi_{2}-\varphi_{1}\right)$ throughout the fibreoptic cable. That is even true before the photons are emitted.

The light cones that are published in articles about the Bell test experiments are wrong. The question is: When do you start the stopwatch? TEW says the stopwatch starts long before the particles are emitted. The field may have been established last week, which gives TEW a 10,080 second head start, meaning that the minimum width of a light cone could be $3 \times 10^{9}$ kilometers.

\subsection{Bell's inequality}

In many experiments coincidence rate $=\sin ^{2} \theta$ is used to test for a violation of Bell's inequality. If we rotate the axes, the coincidence rate of $\cos ^{2} \theta$ also gives us results that also violate Bell's inequality. One experiment found a coincidence rate of $\sin ^{2}(\theta+x)$, where the variable " $x$ " varied depending on the time of the day, as the temperature of the equipment changed. That entire family of sinusoidal squared curves violates Bell's inequality. Alain Aspect et.al. reported a coincidence rate of $\cos ^{2} \theta$ in 1982 (Fig. 15). 


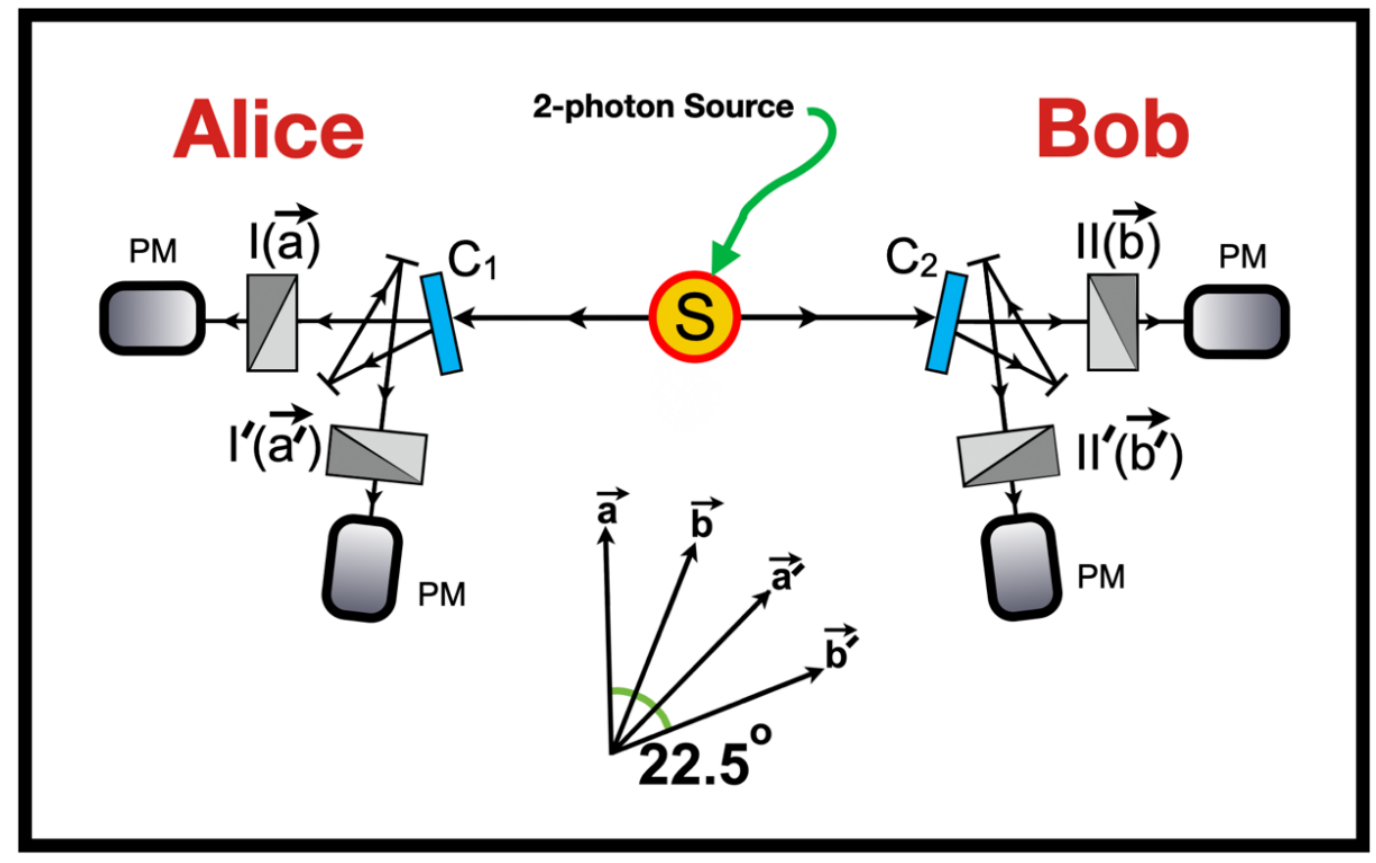

Fig. 15. Equipment used by Aspect, Grangier and Roger in 1982. Entangled photons departed the source " $S^{\prime \prime}$ and were assigned by a randomizing device $\left(C_{1}\right.$ or $C_{2}$ shown in blue) to Wollaston prisms angled at $22.5^{\circ}$ increments, and then observed by photomultipliers (PM). The angles of measurement were chosen to maximize the discrepancies between QM and Einstein's predictions.

In TEW a pair of entangled photons start in the center, halfway between Alice and Bob, and each photon follows the same Bi-Ray in opposite directions (Fig. 16). Our boundary condition $\left(\partial \Omega_{\mathbb{E} \in \mathbb{E}}\right)$ defines the probability of one photon following a Bi-Ray as the amplitude of it following one ray times the amplitude of it following the countervailing ray.

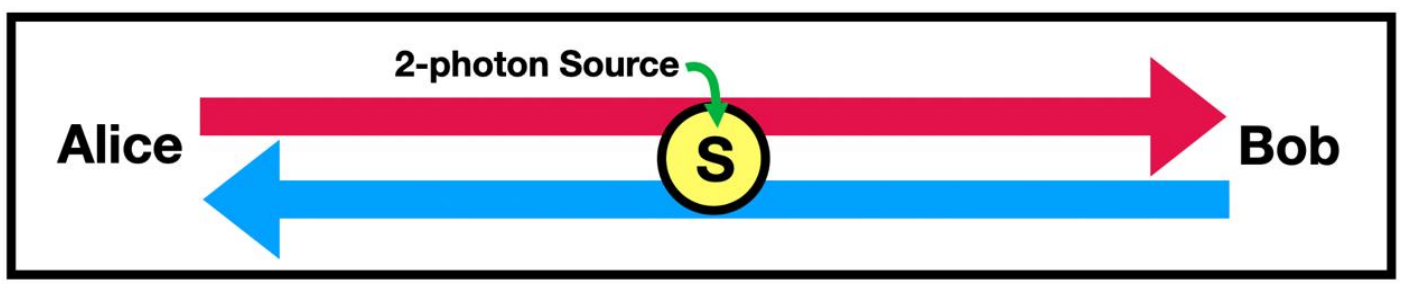

Fig. 16. An elementary ray (red) from Alice travels through the photon Source and reaches Bob's equipment and vice-versa.

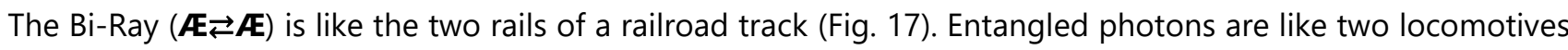
travelling in opposite directions on the same railroad track. All the energy comes from the locomotives, none from the track. The Bi-Ray is established long before photons are emitted. 


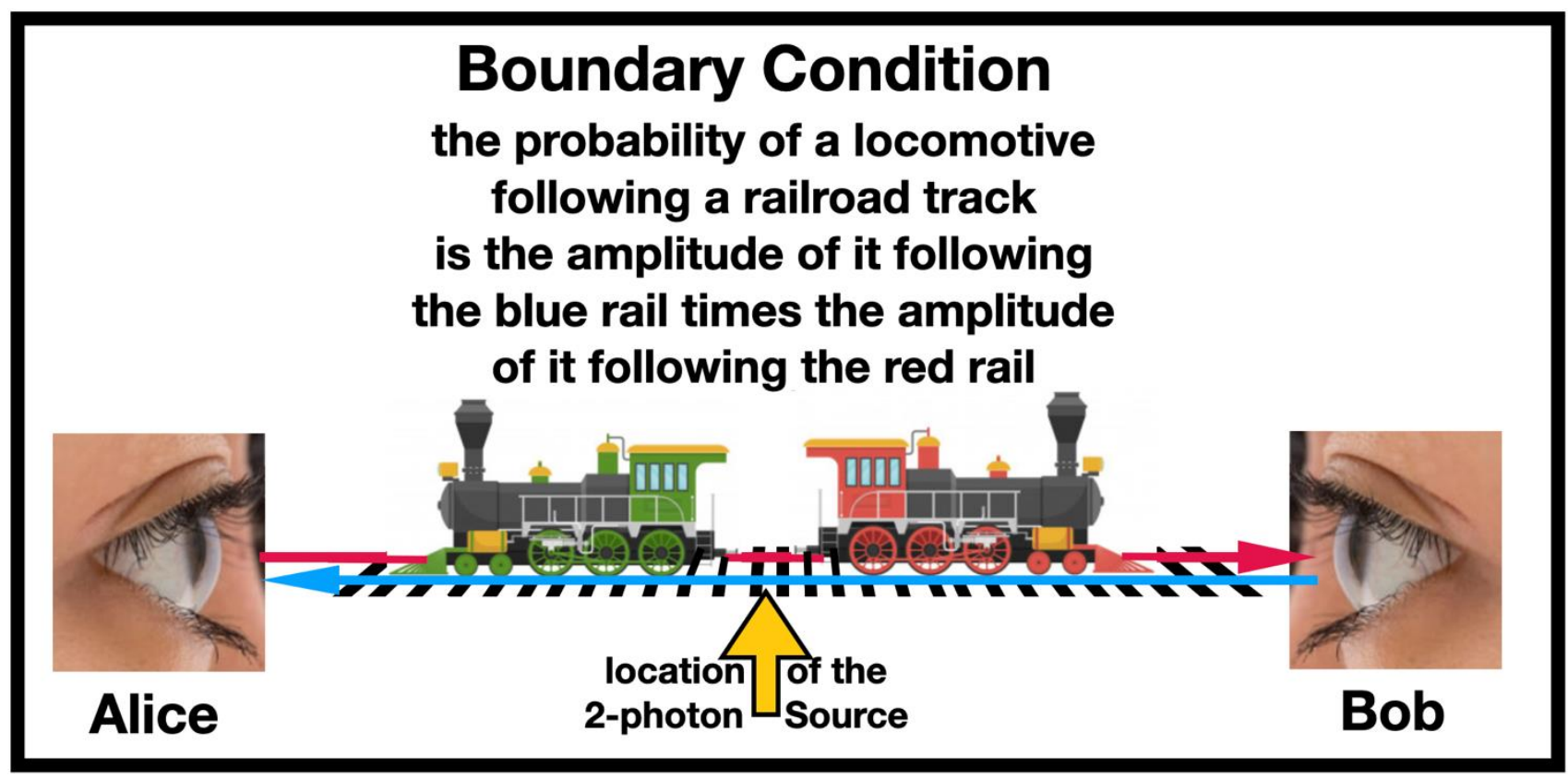

Fig. 17. Here we use a locomotive to represent each of the photons. This boundary condition is the only assumption that we need to explain the Bell test experimental results.

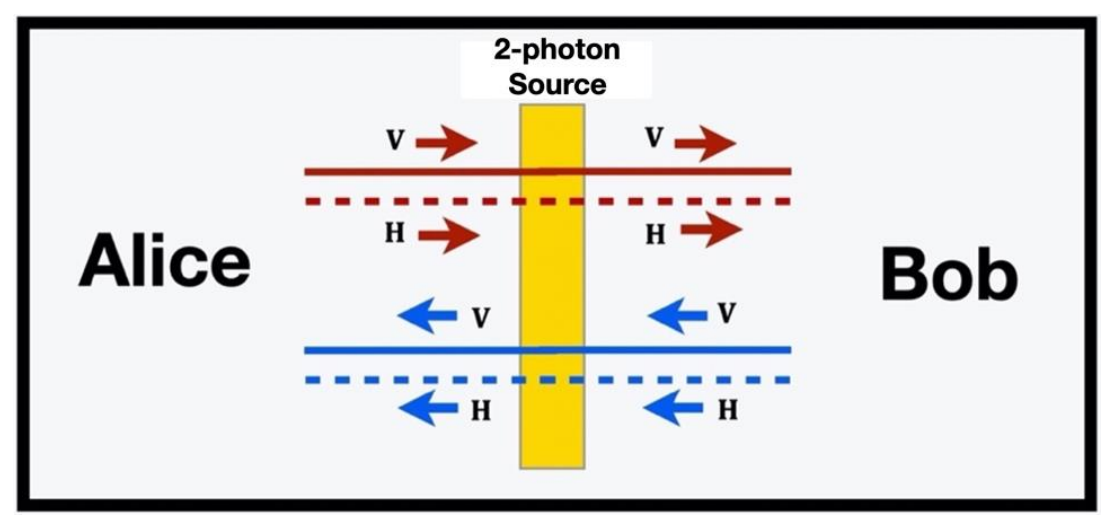

Fig. 18. Using vertical (solid lines) and horizontal (dashed lines) polarized elementary waves, we re-draw Fig. 16. We will use red to denote an elementary ray $(\boldsymbol{A})$ travelling to the right, and blue for an $\boldsymbol{A}$ travelling to the left. Note that these polarized waves above apply only to the individual elementary rays. In the Fig. 19 the four Bi-Ray $(\boldsymbol{A} \rightleftarrows \boldsymbol{E})$ eigenstates are given. 


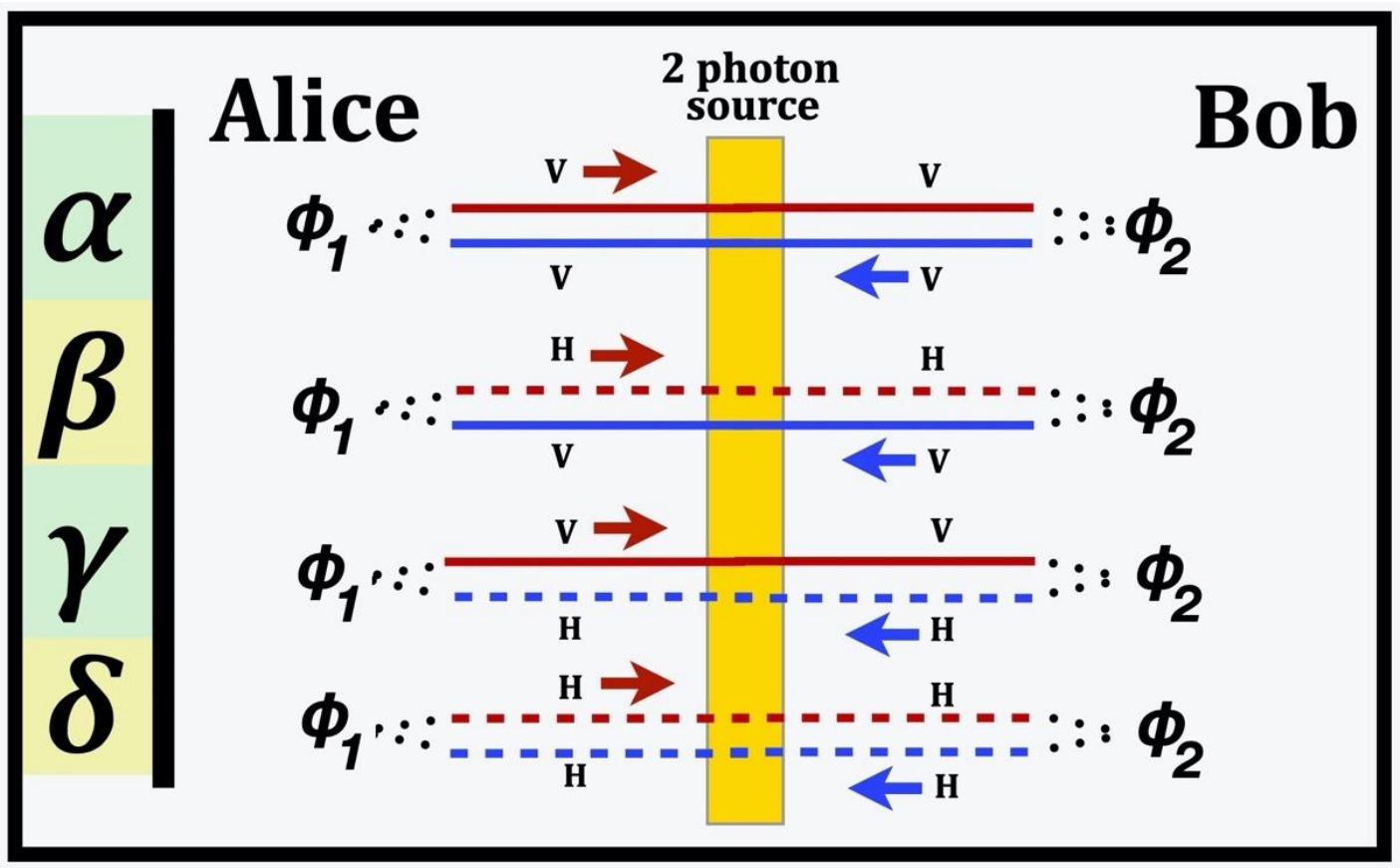

Fig. 19. We define four new eigenstates $(\boldsymbol{\alpha}, \boldsymbol{\beta}, \boldsymbol{\gamma}$ and $\boldsymbol{\delta}$ ) of the Bi-Ray $\boldsymbol{A} \rightleftarrows \boldsymbol{E}$ stretching between Alice and Bob. The angles $\varphi_{1}$ and $\varphi_{2}$ are the random angles of Alice and Bob's polarizers. Neither Alice nor Bob influence or even know what angle of polarization the other person is using. The probability of both Alice and Bob simultaneously seeing a photon (the so-called "coincidence rate") in the $\boldsymbol{\alpha}$ eigenstate is the probability of Alice seeing a photon $\left(\sin \left(\varphi_{1}\right) \sin \left(\varphi_{1}\right)\right)$ times the probability of Bob seeing a photon $\left(\sin \left(\varphi_{2}\right) \sin \left(\varphi_{2}\right)\right)$. This becomes the first line of our equation
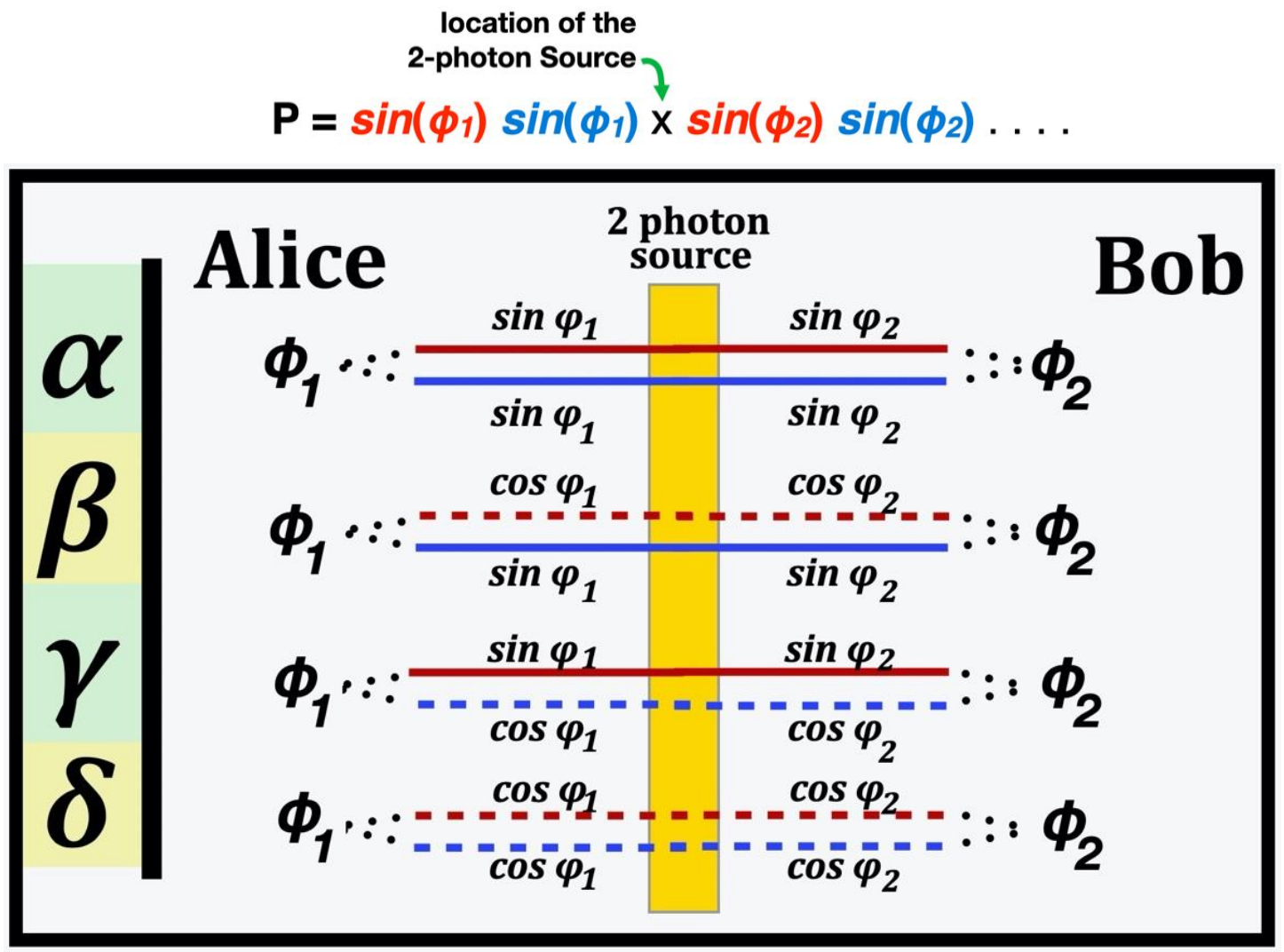

Fig. 20. These sines and cosines show the amplitude for a photon from the source being detected by Alice or Bob in a specific eigenstate when their polarizers have been set to angles $\varphi_{1}$ and $\varphi_{2}$ respectively. Note that 
one photon travels to the left and the other photon to the right. Alice's choice of angle $\varphi_{1}$ is independent of Bob's choice of angle $\varphi_{2}$. Alice detects a different photon than does Bob.

To find the probability of Alice and Bob simultaneously seeing a photon (called the "coincidence rate"), we add together the four eigenstates $(\boldsymbol{\alpha}, \boldsymbol{\beta}, \boldsymbol{\gamma}$ and $\boldsymbol{\delta})$ in Fig. 20:

$\mathbf{P}=\sin \left(\varphi_{1}\right) \sin \left(\varphi_{1}\right) \times \sin \left(\varphi_{2}\right) \sin \left(\varphi_{2}\right) \leftarrow($ within eigenstate $\alpha)$

$+\cos \left(\varphi_{1}\right) \sin \left(\varphi_{1}\right) \times \cos \left(\varphi_{2}\right) \sin \left(\varphi_{2}\right) \leftarrow($ within eigenstate $\beta)$

$+\sin \left(\varphi_{1}\right) \cos \left(\varphi_{1}\right) \times \sin \left(\varphi_{2}\right) \cos \left(\varphi_{2}\right) \leftarrow($ within eigenstate $\boldsymbol{\gamma})$

$+\cos \left(\varphi_{1}\right) \cos \left(\varphi_{1}\right) \times \cos \left(\varphi_{2}\right) \cos \left(\varphi_{2}\right) \leftarrow$ (within eigenstate $\left.\delta\right)$

When we add those four lines together, the result can be factored:

$$
\begin{aligned}
& =\left[\sin \left(\varphi_{1}\right) \sin \left(\varphi_{2}\right)+\cos \left(\varphi_{1}\right) \cos \left(\varphi_{2}\right)\right] \\
& x\left[\sin \left(\varphi_{1}\right) \sin \left(\varphi_{2}\right)+\cos \left(\varphi_{1}\right) \cos \left(\varphi_{2}\right)\right]
\end{aligned}
$$

There is a trigonometry relationship that allows us to compress those two lines into two functions:

$$
\begin{aligned}
& =\cos \left(\varphi_{2}-\varphi_{1}\right) \times \cos \left(\varphi_{2}-\varphi_{1}\right) \\
& =\text { "coincidence rate" }=\cos ^{2}\left(\varphi_{2}-\varphi_{1}\right)
\end{aligned}
$$

This is how TEW accounts for the Bell test data. The math is astonishingly simple. Our only starting assumption was that a pair of entangled photons follows a Bi-Ray: $\boldsymbol{A E} \rightleftarrows \boldsymbol{E}$. Our prediction is that the coincidence rate will be $\mathbf{P}=\cos ^{2}\left(\varphi_{2}-\varphi_{1}\right)$. If the 2-photon-Source were changed so it emitted photons orthogonal to one another, then the final coincidence rate would be

$$
P=\sin ^{2}\left(\varphi_{2}-\varphi_{1}\right) \text {. }
$$

TEW shows how the quantum math stretches across space without exceeding the lightspeed limit. TEW obeys the speed-of-light rules but starts earlier than photon emission and therefore appears to go faster than lightspeed. By the time a photon pair is emitted, Bi-Rays are long since established. Wave function collapse (consisting of each entangled photon attaching to the same Bi-Ray) occurs as the photons are emitted and not when the photons are measured by Alice and Bob's detectors. But the Bi-Ray is established even before 2photon emission.

Einstein thought each particle had solid characteristics (hidden variables) even before it was observed. TEW says that is wrong. It is not the intrinsic characteristics of particles, but the elementary waves that constitute the canvas upon which reality is painted. The characteristics of each particle depend on which angle it is observed at. Each person who asks a different question, will get a different answer.

When QM sees two entangled particles in a Bell test experiment performing a choreographed dance, they invoke the doctrine of "nonlocality" because there is no mechanism for that choreography to reach across empty space. "Nonlocality" is a vague term, which has no scientific merit: it is so vague that it does not inspire experiments to investigate it.

As we said before, the Bi-Ray model of TEW provides a domain that spans the entire fiberoptic cable.

\section{Conclusion}

The biggest accomplishment of this article is to demonstrate that, within the fanciful world of our mathematical games, quantum weirdness can be solved as a mathematical problem.

In this article we presented two games. In the first (the "elementary wave game") all weirdness was concentrated into one single point named $\boldsymbol{\alpha}$. We introduced you into a fanciful world in which that point could be understood if and only if you solved the problem of quantum weirdness everywhere. Although this was presented as a game or machine, we believe it is the first time that anyone has proposed a mathematical solution to the problem of quantum weirdness. 
In the second game, we proposed a non-Einstein, non-QM interpretation of the Bell test experiments.

As we said before, this is not another "interpretation" of QM. It is a different mathematics.

\subsection{YouTube video}

Associated with this article is a YouTube video published in 2021 with the title "PDE boundary conditions that eliminate quantum weirdness." You can find it by typing that title into the search engine of YouTube. You can also find it by going to my YouTube channel "elwavetheory". YouTube will redirect you to "wave theory" instead, and you have to remind YouTube you want to go to "elwavetheory". That video is one hour in length and is a fun way to understand this article from a different perspective.

\section{Acknowledgement}

The author thanks Lewis E. Little.

\section{Conflict of interest}

The author declares that there is no conflict of interest regarding the publication of this article.

\section{Author Biography}

The author lives in Connecticut, USA. He has graduated with diplomas from Harvard, Yale, Brown, and Case Western Reserve Universities and has been trained in mathematics, more than one science, and more than one spiritual tradition. As of 2021 he retired.

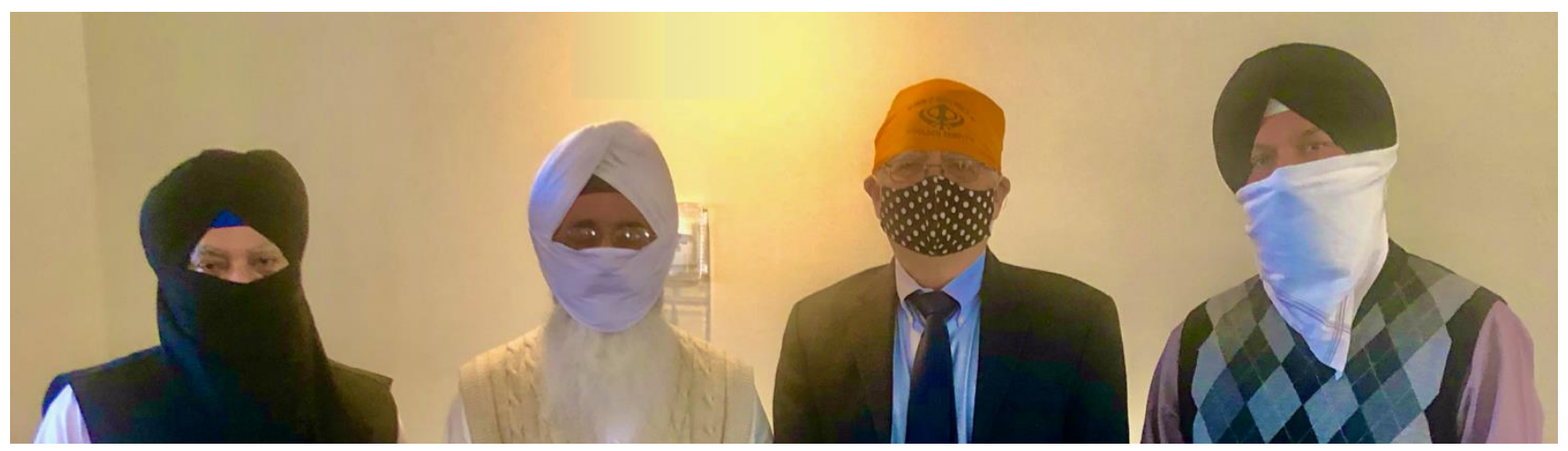

Jeffrey Boyd is the second from right, at Gurudwara Sachkhand Darbar, Hamden, CT.

Everyone is masked because of the COVID-19 pandemic.

This article is the product of five decades of work: four by Lewis E. Little (the author's cousin) and one decade by this author. Recently, preparing to write this article, this author invented the PDE model contained in this article.

The author believes that there is a contradiction embedded into the very structure of physical reality. The bottom line is that things are not what they appear to be. This is consistent with the Sanskrit word māyā; which means that what appears to be true is actually an illusion:

\section{माया}

\section{References}

1. J. Baggott, The Quantum Story: a history in 40 moments, Oxford University Press, 2011. ISBN:978-0-19956684-6

2. A. Bassi, K. Lochan, S. Satin, T.P. Singh and H. Ulbricht, "Models of wave function collapse," Reviews of Modern Physics, 85. 471. DOI: 10.1103/RevModPhys.85.471

3. A. Becker, What Is Real? Basic Books, 2018. ISBN:978-0-19-956684-6 
4. M. Born, "On the quantum mechanics of collisions," in J. A. Wheeler and W. H. Zurek (eds.), Quantum Theory and Measurement, Princeton, pp.50-55, 1983. ISBN 978-0-691-08316-2.

5. J. H. Boyd, "Six reasons to discard wave particle duality." Journal of Advances in Chemistry, 18, 1-29, 2021. DOI: $10.24297 / j a c . v 18 i .8948$

6. J. H. Boyd, "6 reasons to discard wave particle duality," a YouTube video, 2021, at https://www.youtube.com/watch?v=LD4xXI5gMzg\&t=2s.

7. J. H. Boyd, "The Periodic Table needs negative orbitals in order to eliminate quantum weirdness," Journal of Advances in Chemistry, vol. 17, pp.88-125, 2020. DOI: 10.24297/jac.v17i.8865

8. J. H. Boyd, "There are two solutions to the equations of Feynman's Quantum Electrodynamics (QED); the newly discovered solution is free of quantum weirdness," Journal of Advances in Physics, vol. 18, pp. 3957, 2020. DOI: 10.24297/jap.v18i.8831.

9. J. H. Boyd, "If the propagator of QED were reversed, the mathematics of Nature would be much simpler," Journal of Advances in Mathematics, vol. 18, pp. 129-153, 2020. DOI: 10.24297/jam.v18i.8746

10. J. H. Boyd, "A tiny, counterintuitive change to the mathematics of the Schrödinger wave packet and Quantum ElectroDynamics could vastly simplify how we view Nature," Journal of Advances in Physics, vol. 17, pp. 169-203, 2020. DOI: 10.24297/jap.v17i.8696

11. J. H. Boyd, "New Schrödinger wave mathematics changes experiments from saying there is, to denying there is quantum weirdness," Journal of Advances in Mathematics, vol. 18, pp. 82-117, 2020. DOI: 10.24297/jap.v17i.8696

12. J. H. Boyd, "Decrypting the central mystery of quantum mathematics: Part 1. The double slit experiment," Journal of Advances in Mathematics, 2 vol. 17, pp. 255-282, 2019. DOI: 10.24297/jam.v17i0.8475

13. J. H. Boyd, "Decrypting the Central Mystery of Quantum Mathematics: Part 2. A mountain of empirical data supports TEW," Journal of Advances in Mathematics, vol. 17, pp. 283-314, 2019. DOI: 10.24297/jam.v17i0.8489

14. J. H. Boyd, "Decrypting the central mystery of quantum mathematics: Part 3. A non-Einstein, non-QM view of Bell test experiments," Journal of Advances in Mathematics, vol. 17, pp. 315-331, 2019. DOI: 10.24297/jam.v17i0.8490

15. J. H. Boyd, "Decrypting the central mystery of quantum mathematics: Part 4. In what medium do Elementary Waves travel?" Journal of Advances in Mathematics, vol. 17, pp. 332-351, 2019. DOI: 10.24297/jam.v17i0.8491

16. J. H. Boyd, "The quantum world is astonishingly similar to our world," Journal of Advances in Physics, vol. 14, pp. 5598-5610, 2018. DOI: 10.24297/jap.v14i2.7555

17. J. H. Boyd, "The von Neumann and double slit paradoxes lead to a new Schrödinger wave mathematics," Journal of Advances in Physics, vol.14, pp. 5812-5834, 2018. doi.org/10.24297/jap.v14i3.7820

18. J. H. Boyd, "The Boyd Conjecture," Journal of Advances in Physics, vol. 13, pp. 4830-4837, 2017. DOI: 10.24297/jap.v13i4.6038

19. J. H. Boyd, "A symmetry hidden at the center of quantum mathematics causes a disconnect between quantum math and quantum mechanics," Journal of Advances in Mathematics, vol. 13, pp. 7379-7386, 2017. DOI: $10.24297 / j a m . v 13 i 4.6413$

20. J. H. Boyd, "Paul Dirac's view of the Theory of Elementary Waves," Journal of Advances in Physics, vol. 13, pp. 4731-4734, 2017. DOI: 10.24297/jap.v13i3.5921

21. J. H. Boyd, "A paradigm shift in mathematical physics, Part 1: The Theory of Elementary Waves (TEW)," Journal of Advances in Mathematics, vol. 10, pp. 3828-3839, 2015. DOI: 10.24297/jam.v10i9.1908 
22. J. H. Boyd, "A paradigm shift in mathematical physics, Part 2: A new local realism explains Bell test \& other experiments," Journal of Advances in Mathematics, vol. 10, pp. 3828-3839, 2015. DOI: 10.24297/jam.v10i9.1884

23. J. H. Boyd, "A paradigm shift in mathematical physics, Part 3: A mirror image of Feynman's quantum electrodynamics (QED)," Journal of Advances in Mathematics, vol. 11, pp. 3977-3991, 2015. DOI: 10.24297/jam.v11i2.1283

24. J. H. Boyd, "A paradigm shift in mathematical physics, Part 4: Quantum computers and the local realism of all 4 Bell states," Journal of Advances in Mathematics, vol. 11, pp. 5476-5493, 2015. DOI: 10.24297/jam.v11i7.1224

25. J. H. Boyd, "The Theory of Elementary Waves eliminates Wave Particle Duality," Journal of Advances in Physics, vol. 7, pp. 1916-1922, 2015. DOI: 10.24297/jap.v7i3.1576

26. J. H. Boyd, "A new variety of local realism explains a Bell test experiment," Journal of Advances in Physics, vol. 8, pp. 2051-2058, 2015. DOI: 10.24297/jap.v8i1.1541

27. J. H. Boyd, "A proposed physical analog of a quantum amplitude," Journal of Advances in Physics, vol. 10, pp. 2774-2783, 2015. DOI: 10.24297/jap.v10i3.1324

28. J. H. Boyd, "Re-thinking a delayed choice quantum eraser experiment: a simple baseball model," Physics Essays, vol. 26, pp. 100-109, 2013. DOI: 10.4006/0836-1398-26.1.100

29. J. H. Boyd, "Re-thinking Alain Aspect's 1982 Bell test experiment with delayed choice," Physics Essays, vol. 26, pp. 582-591, 2013. DOI: 10.4006/0836-1398-26.1.100 10.4006/0836-1398-26.4.582

30. J. H. Boyd, "Rethinking a Wheeler delayed choice gedanken experiment," Physics Essays, vol. 25, pp. 390396, 2012. DOI: 10.4006/0836-1398-25.3.390

31. B. Carlson, "Boundary conditions in the time independent Schrödinger equation," 2013, https://www.youtube.com/watch?v=dWDJIMjWxIM

32. J. F. Clauser, M. A. Horne, A. Shimony and R. A. Holt, "Proposed experiment to test local hidden-variable theories." Physical Review Letters 23, 880-884, 1969. DOI: 10.1103/PhysRevLett.23.880

33. L. de Broglie, Research on the Theory of Quanta, translated by André Michaud and Fritz Lewertoff, Montreal: Minkowski Institute Press, 2021. ISBN: 1-927763-99-5.

34. H. Everett, J. A. Wheeler, B.S. DeWitt, L.N.Cooper, D. Van Vechten, and N. Graham, "Theory of the Universal Wave-Function," pp. 1-150, in DeWitt, B.S., and N. Graham, editors, The Many Worlds Interpretation of Quantum Mechanics, Princeton University Press (1973). ISBN: 0-691-88131-X

35. C. A. Fuchs, N. D. Mermin, and R. Schack, "An introduction to QBism with an application to the locality of quantum mechanics," arXiv: 1311.52531v1, November 20, 2013.

36. C. A. Fuchs and A. Peres, "Quantum theory needs no 'interpretation,'" Physics Today, vol. 53, issue 3, p. 70 (March 2000). DOI: 10.1063/1.88304

37. K. Gödel, "The incompleteness theorem," in S. Hawkings, editor, 2005. God Created the Integers, Running Press, Philadelphia, ISBN 0-7624-1922-9. Gödel's paper appears starting on p. 1097

38. A. Harris, "Animating Schrödinger's equation." http://https//www.youtube.com/watch?v=cV2fkDscwvY

39. W. Heisenberg, The Physical Principles of the Quantum Theory, translated by Carl Eckart and Frank C. Hoyt Dover Publications. Library of Congress Number 49-11952.

40. D. Hilbert and W. Ackermann, The Foundations of Mathematical Logic, translated by L. M. Hammond, G. G. Leckie, and R.E. Luce, AMS Chelsea Publishing, Providence R.I., USA, (c1958) ISBN-13: 978-0821820247

41. R. G. Hulet, E. S. Hilfer, and D. Kleppner, "Inhibited spontaneous emission by a Rydberg atom," Physical Review Letters, vol. 55, pp. 2137-2140, 1985. https://doi.org/10.1103/PhysRevLett.55.2137 
42. H. Kaiser, R. Clothier, S. Werner, et. al., "Coherence and spectral filtering in neutron interferometry," Physical Review A, vol. 45, pp. 31-42, 1992. DOI: 10.1103/PhysRevA.45.31

43. T. S. Kuhn, The Structure of Scientific Revolutions, (Chicago: U. of Chicago Press, 1970. ISBN 978-0-22645803-8.

44. L. E. Little, The Theory of Elementary Waves, (New Classics Library, Gainsville, GA), 2009. ISBN: 978-0932750-84-6.

45. L. E. Little, "Theory of Elementary Waves," Physics Essays, vol. 9, pp. 100-134, 1996. DOI: 10.4006/1.3029212

46. L. E. Little, "Theory of Elementary Waves @ JPL, Feb 2000," https://www.youtube.com/watch?v=3_9LBORzgWg

47. L. E. Little "Introduction to Elementary Waves," 2016. https://www.youtube.com/watch?v=xx5V03iCbAo\&t=16s

48. L. E. Little, "We have seen these waves," 2016. https://www.youtube.com/watch?v=xWMiNsD_xdM\&t=5s

49. N. D. Mermin, "Is the moon there when nobody looks? Reality and the quantum theory," Physics Today, 38, 38-47 (1985). DOI: 10.1063/1.880968

50. N. D. Mermin, "'Measured responses to quantum Bayesianism," Physics Today, 65 (12) (December 2012), pp. 12-13. DOI: 10.1063/PT.3.1803.

51. J. R. Pierce, Almost All About Waves, New York: Dover Books, 1974. ISBN: 0-486-45302-2.

52. E. M. Purcell, "Proceedings of the American Physical Society: Spontaneous Emission Probabilities at Ratio Frequencies" Physical Review. American Physical Society 69 (11-12): 681. http://pages.erau.edu/ reynodb2/colloquia/Purcell_1946_SpontaneousEmission.pdf

53. H. Schlichtkrull, "Boundary value problems for partial differential equations," 2013,

a. $\quad$ http://web.math.ku.dk/ schlicht/DL/2013/PDE13.pdf

54. E. Schrödinger, Collected Papers on Wave Mechanics, Montreal: Minkowski Institute Press, 2020, ISBN: $978-$ 1-927763-81-0.

55. E. Schrödinger, Abhandlunen zur Wellenmechanik, published by Leipzig: Johann Ambrose Barth, 1928.

56. J. C. Slater, "Physics and the Wave Equation," the Josiah Willard Gibbs lecture, at the American Mathematical Society and the Mathematical Association of America, in Chicago, IL, USA Nov 23, 1946

a. $\quad$ https://www.ams.org/journals/bull/1946-52-05/S0002-9904-1946-08558-4/S0002-9904-1946-085584.pdf

57. A. Turing, "On computable numbers, with an application to the Entscheidungsproblem," Proceedings of the London Mathematical Society, Volume s2-42, Issue 1, 1937, Pages 230-265, DOI: 10.1112/plms/s242.1.230

58. ViaScience, "Bell's inequality II," YouTube, https://www.youtube.com/watch?v=8UxYKN1q5s/\&t=3s

59. J. von Neumann, Mathematical Foundations of Quantum Mechanics, translated by Robert T. Beyer, (Princeton NJ: Princeton University Press, c1955). ISBN: 0-691-02893-1

60. S. A. Werner, R. Clothier, H. Kaiser, et.al., "Spectral filtering in neutron interferometry," Physical Review Letters, vol. 67, pp.683-686, 1991. DOI: 10.1103/PhysRevLett.67.683 